\title{
Bottom Boundary Potential Vorticity Injection from an Oscillating Flow: A PV Pump
}

\author{
XiAOZHOU RuAN AND ANDREW F. THOMPSON \\ Environmental Science and Engineering, California Institute of Technology, Pasadena, California
}

(Manuscript received 18 November 2015, in final form 1 September 2016)

\begin{abstract}
Oceanic boundary currents over the continental slope exhibit variability with a range of time scales. Numerical studies of steady, along-slope currents over a sloping bathymetry have shown that cross-slope Ekman transport can advect buoyancy surfaces in a bottom boundary layer (BBL) so as to produce vertically sheared geostrophic flows that bring the total flow to rest: a process known as buoyancy shutdown of Ekman transport or Ekman arrest. This study considers the generation and evolution of near-bottom flows due to a barotropic, oscillating, and laterally sheared flow over a slope. The sensitivity of the boundary circulation to changes in oscillation frequency $\omega$, background flow amplitude, bottom slope, and background stratification is explored. When $\omega / f \ll 1$, where $f$ is the Coriolis frequency, oscillations allow the system to escape from the steady buoyancy shutdown scenario. The BBL is responsible for generating a secondary overturning circulation that produces vertical velocities that, combined with the potential vorticity (PV) anomalies of the imposed barotropic flow, give rise to a time-mean, rectified, vertical eddy PV flux into the ocean interior: a "PV pump." In these idealized simulations, the PV anomalies in the BBL make a secondary contribution to the timeaveraged PV flux. Numerical results show the domain-averaged eddy PV flux increases nonlinearly with $\omega$ with a peak near the inertial frequency, followed by a sharp decay for $\omega / f>1$. Different physical mechanisms are discussed that could give rise to the temporal variability of boundary currents.
\end{abstract}

\section{Introduction}

Boundary processes exert a strong influence over large-scale circulation patterns and energy budgets in both the ocean and the atmosphere (Wunsch 1970; Schneider et al. 2003). In the ocean, boundary currents over continental shelves and slopes can influence water mass modification (Whitworth et al. 1998; Orsi et al. 2002; Flexas et al. 2015), eddy formation (Molemaker et al. 2015; Gula et al. 2015), and the generation of potential vorticity (PV) anomalies (Williams and Roussenov 2003). In coastal regions, interactions between ocean boundaries and mean flows influence the distribution of nutrients and other biogeochemical tracers (Gruber et al. 2006; Dulaiova et al. 2009).

The variability associated with these boundary currents is also critical for modifying both buoyancy distributions and turbulent properties in the ocean's

Corresponding author address: Xiaozhou Ruan, Environmental Science and Engineering, MC 131-24, California Institute of Technology, Pasadena, CA 91125.

E-mail: xiaozhour@caltech.edu bottom boundary layer (BBL). Moum et al. (2004) showed, off the coast of Oregon, that as the orientation (sign) of the along-slope flow changes, the advection of buoyancy surfaces related to either Ekman upwelling or Ekman downwelling can result in quite different turbulent kinetic energy states in the bottom boundary layer. These transitions indicate that there is a coupling between along-slope mean flows and boundary layer circulations over sloping bottom boundaries. Additionally, numerical studies have shown that flow separation over sloping bottom boundaries can be responsible for the generation of mesoscale eddies and their transport into the ocean gyres (e.g., Molemaker et al. 2015). Thus, the dynamics of boundary layers are likely to impact various aspects of the ocean circulation, such as the along-slope front variability, eddy generation, water mass modification, and ventilation near continental slopes and shelves.

Part of the motivation for this study comes from recent observations of high-latitude boundary currents in both the Arctic and Antarctic. Spall et al. (2008) showed that narrow, bottom-intensified currents in the Beaufort Gyre give rise to PV anomalies over a limited vertical 
extent above the continental slope. In numerical simulations, these anomalies are shown to be stirred into the interior, leading to a banded structure in PV anomalies far from the boundary. During a glider field experiment in the western Weddell Sea, Thompson et al. (2014) observed similar banded PV distributions over the continental slope believed to come from interactions of a predominantly barotropic mean flow generated by the surface wind stress and the sloping boundary. These PV gradients are linked to the Antarctic marginal overturning circulation, which helps to bring the warm middepth water over the shelf break toward the ice shelf grounding line. In another numerical experiment, tidal forcing is shown to be critical in reproducing the crossslope structure and time variability of the Antarctic Slope Front (ASF) and Antarctic Slope Current (ASC) along the South Scotia Ridge (Flexas et al. 2015). This study showed that even without atmospheric forcing, tides can generate the observed structure and variability of the ASF and ASC through tidal rectification over the continental slope. This study suggests that oscillating boundary currents impact the large-scale background density and PV fields.

While there exists a substantial literature on BBL dynamics, understanding of the physical processes that dominate the BBL evolution remains incomplete due to the difficulty of observing and simulating these turbulent processes. This has also led to diverse approaches in representing BBL turbulence. One approach has been direct numerical simulation or large-eddy simulations of the boundary layer (Gayen et al. 2010; Gayen and Sarkar 2011). These simulations are typically limited to relatively small domains that are unable to capture the mechanisms that are the focus of this paper, associated with a jet of width $\sim 10 \mathrm{~km}$ and will not be discussed further here. Two other approaches have commonly been used to treat the boundary layer turbulence: (i) a "laminar" relationship where the stress is related to a viscosity and a velocity shear, noting that this is an eddy viscosity, which encapsulates the turbulent dynamics (MacCready and Rhines 1991), and (ii) a turbulent quadratic parameterization (Brink and Lentz 2010a).

A major result of the first, laminar path has been the mechanistic identification and explanation of Ekman arrest or the buoyancy shutdown of Ekman transport. Thorpe (1987) first developed an analytical model of the adjustment of density surfaces in response to a mean flow over a sloping bottom, which provided steady-state solutions for along-slope and cross-slope velocities. Subsequently, a series of studies showed that the influence of the boundary layer on the interior could be reduced due to Ekman arrest (MacCready and Rhines 1991; Trowbridge and Lentz 1991; Chapman 2002). As a background mean flow interacts with a sloping bottom, a frictional Ekman boundary layer will develop within an inertial time scale. These Ekman velocities advect buoyancy surfaces and produce horizontal buoyancy gradients that balance vertically sheared geostrophic velocities (thermal wind balance) that oppose the mean flow (Fig. 1a). This adjustment mainly happens in a thermal boundary layer defined by the influence of buoyancy diffusion. The result of this Ekman arrest is that both the bottom stress and cross-slope Ekman transport are weakened. Even in the absence of a background flow, isopycnals tend to tilt downslope to meet the no-flux boundary condition, and the resulting horizontal buoyancy gradient generates along-slope velocities and a corresponding cross-slope Ekman transport. This is called the buoyancy generation of Ekman transport. The time scale for buoyancy shutdown and buoyancy generation processes are similar; a scaling is provided below.

The second, turbulent parameterization path has mostly followed the work by Trowbridge and Lentz (1991). Following this line, Brink and Lentz examined the buoyancy arrest in a turbulent boundary layer in response to a steady mean flow (Brink and Lentz 2010a) and a 1D oscillatory flow (Brink and Lentz 2010b). Differences in the arrest time scale between bottom Ekman upwelling and downwelling cases were identified; these arise from the turbulent mixing parameterization. This study also showed that in the upwelling case, the boundary layer has a more complicated vertical structure, which may include a "transition" layer between the boundary layer and the ocean interior. These cases were referred to as the "smooth upwelling" and "capped upwelling." While Ekman arrest has also been studied and confirmed using the turbulent parameterization, the mechanism by which the interior flow is damped is not as clearly identified in the momentum equation.

Despite the apparently robust Ekman arrest behavior in numerical simulations, there is limited direct observational evidence of buoyancy shutdown. Furthermore, oceanic observations of the BBL show characteristics of both the laminar and turbulent models. Measurements from moored arrays, in the BBL, collected near the California coast (Trowbridge and Lentz 1998) and the Oregon coast (Perlin et al. 2005), indicate that Ekman arrest is not fully achieved in the ocean. Yet, these studies also confirmed that the leading-order momentum balance, as predicted by analytical models like Trowbridge and Lentz (1991), holds in both the alongslope and cross-slope directions. Perlin et al. (2005) also showed that the vertical stratification may contain multiple layers, including "remnant" turbulent layers 

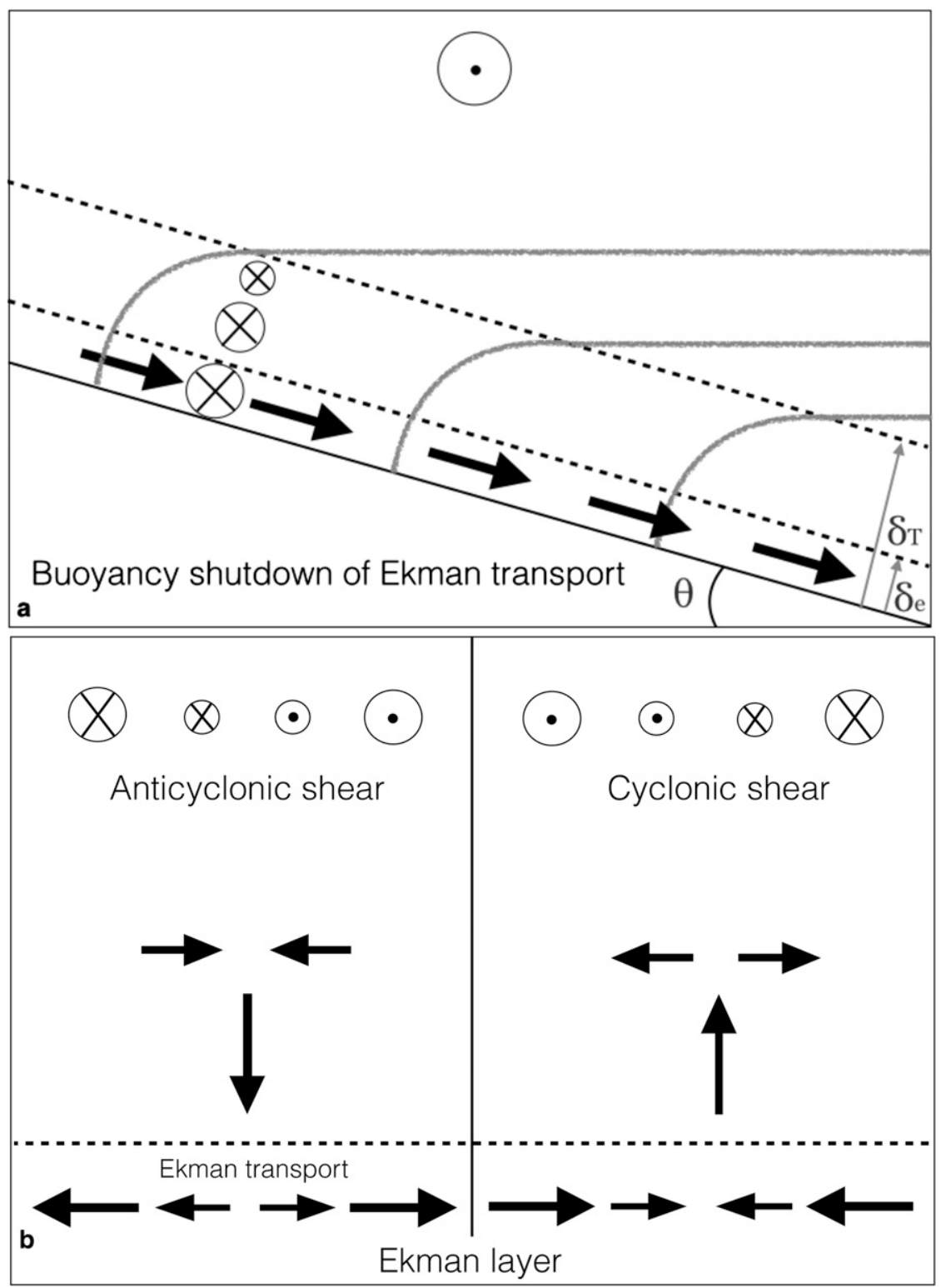

FIG. 1. Schematic representation of bottom boundary layer processes considered in this study: (a) buoyancy shutdown and (b) frictional spindown. Arrows as well as dotted and crossed circles indicate mean flow direction; gray contours are density surfaces and $f>0$. In (a), a laterally uniform mean flow generates an Ekman transport in a boundary layer $\delta_{e}$ that tilts isopycnals, which also diffuse in a boundary layer $\delta_{T}$. The resulting horizontal buoyancy gradient balances a vertically sheared along-slope flow that opposes the interior flow until Ekman transport and buoyancy advection ceases; buoyancy shutdown occurs independent of background flow orientation. In (b), a cyclonic (anticyclonic) circulation over a flat bottom induces Ekman pumping (suction) related to Ekman convergence (divergence). This results in a secondary overturning circulation in opposition to the interior pressure gradient, which depletes the kinetic energy of the background flow.

outside of the BBL. These outer layers evolve on time scales longer than the bottom mixed layer, similar to a thermal diffusive layer, and are likely to influence the interaction between the BBL and the open ocean.
There are many reasons why Ekman arrest may be absent or weak in the ocean. Most simulations predicting Ekman arrest assume two-dimensional dynamics (as we do here); the impact of along-slope variations on 
BBL evolution remain relatively unexplored. Our goal in this study is to understand how a time-dependent mean flow influences Ekman arrest and the general characteristics of the BBL. With a view toward elucidating the physical mechanisms that control this evolution, we adopt an idealized process modeling approach following the eddy viscosity representation of MacCready and Rhines (1991). We acknowledge that verification of the physics discussed here in more complex models is an important next step.

Our approach also follows a series of recent studies that have considered bottom boundary processes in the framework of PV, which is especially useful in eventually assessing the impact of these dynamics on the largerscale ocean circulation (Rhines 1986). Benthuysen and Thomas (2012), hereinafter BT12) developed a onedimensional analytical model that predicts the time evolution of buoyancy and along-isobath velocity in response to a steady mean flow. The results show that the change in the vertically integrated PV depends not only on the mean flow direction, but also on the ratio of the initial mean flow speed to its equilibrated speed. Additionally, the change in the PV is asymmetric: for along-slope currents with the same magnitude but different orientations, removal of PV is more efficient than the injection of PV. A laterally sheared mean flow was introduced by Benthuysen and Thomas (2013, hereinafter BT13), and a two-dimensional analytical model was developed. In this case, along-slope flow induces convergence and divergence of the Ekman transport near the boundary, which drives a vertical secondary circulation that depletes the kinetic energy from the interior via the Coriolis force and lateral momentum advection at both leading order and higher order and decelerates the mean flow (see Fig. 1b). This process is known as nonlinear frictional spindown. Both buoyancy shutdown and frictional spindown may be important if they occur over similar time scales. We adopt the basic setup in BT13 in our study with the addition of a timedependent far-field velocity forcing.

In this study, we explain how a time-dependent barotropic mean flow reduces the efficiency of the buoyancy shutdown state and produces a rectified injection of PV from the BBL into the ocean interior. The vertical PV flux arises from the generation of a secondary overturning circulation in the depth-cross slope plane. In section 2, we introduce the governing equations that give rise to both buoyancy shutdown and frictional spindown. Important parameters and expressions for boundary layer thicknesses are provided. In section 3, we perform nondimensionalization and scaling analyses on the governing equations and identify three physical regimes based on the oscillation frequency: (i) the low-frequency or buoyancy shutdown regime, (ii) the near-inertial regime, and (iii) the high-frequency regime. The dynamics of each regime is described in section 4. In section 5, we address controls on PV anomalies and the flux of PV into the ocean interior. We also explore the sensitivity of the PV flux as a function of oscillation frequency, mean flow amplitude, bottom slope angle, and the background stratification. Discussion of results and conclusions are in section 6 .

\section{The horizontally sheared and oscillating mean flow model}

The equations of motion forced by an oscillating, laterally sheared barotropic flow over a sloping bottom capture both buoyancy shutdown and an interior frictional spindown. Our choice of a barotropic background flow is motivated primarily by observations of strong, narrow (width comparable to the deformation radius) shelfbreak currents found in many regions (Thompson et al. 2014; Woodgate et al. 2001). Critically, the boundary currents are observed to vary over a range of frequencies, as evidenced in Fig. 10 of Thorpe (1987), for example. While these currents may have both barotropic and baroclinic structure, they are largely forced by surface winds that generate sea surface height anomalies through surface Ekman transport. While this forcing will not produce variability with a single frequency, we choose to apply an oscillatory mean flow in order to systematically explore parameter space.

It is important to recognize that this form of the externally forced background barotropic system introduces PV anomalies into the system because of the relative vorticity associated with the current's lateral shear. These PV anomalies integrate to zero over a full oscillation period, and it is only through the interaction with the vertical velocity generated by the secondary circulation that a nonzero PV flux is produced. The generation of the secondary circulation is dependent on the bottom boundary layer dynamics alone. We explored other background flows that do not generate PV anomalies, such as inertial-gravity and Kelvin and topographic Rossby waves, but the dispersion relations for these motions do not reproduce the observed boundary current properties. Our choice of background flow is motivated by choosing a simple system that provides insight into bottom boundary-interior exchange but acknowledge that the results presented here need to be explored in more realistic models, especially those that permit feedbacks between the boundary layer-induced circulation and the interior flow.

The Cartesian coordinate system is rotated with an angle $\theta$ so that the $z$ axis is normal to the slope (Fig. 2). 

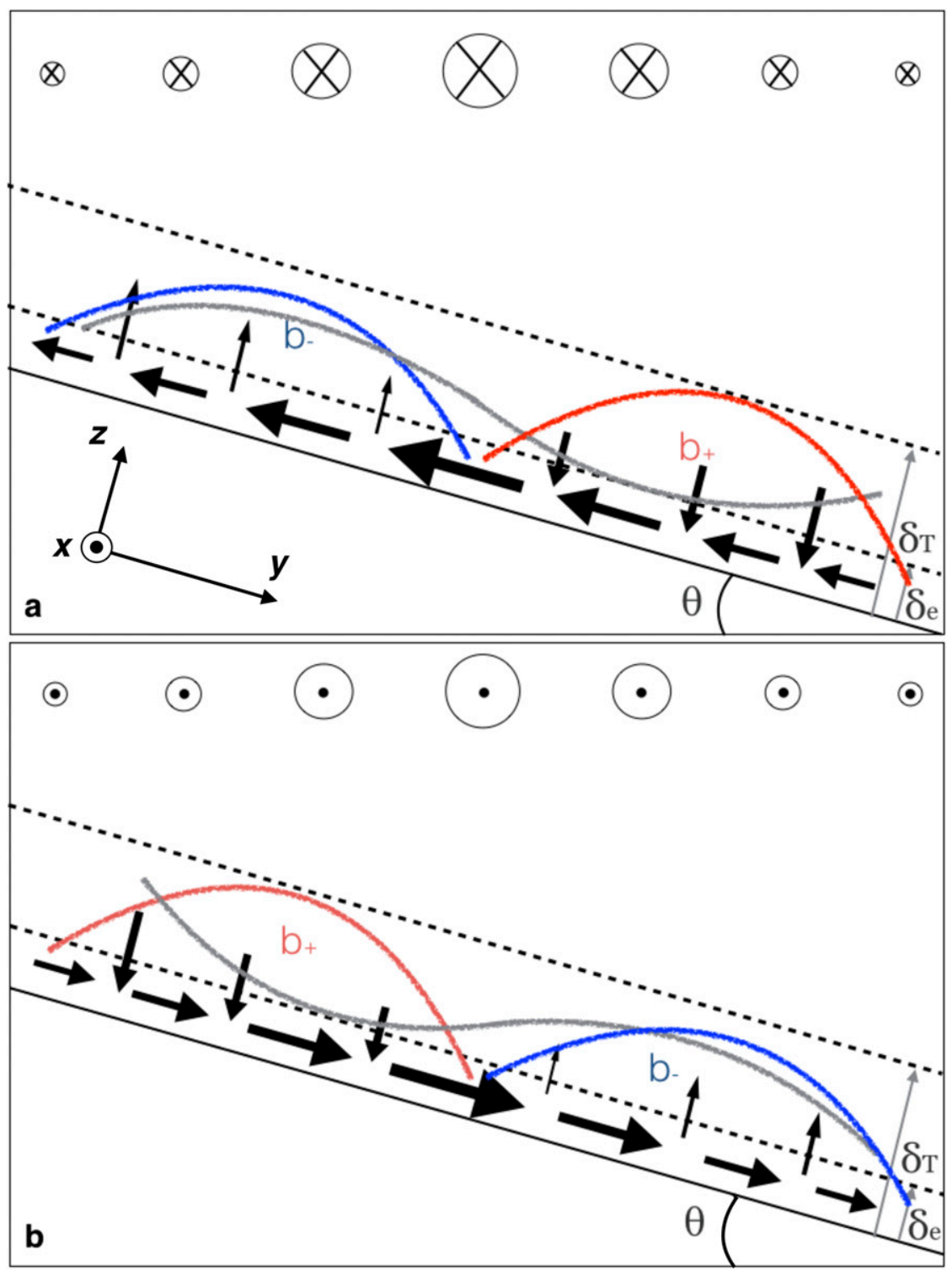

FIG. 2. Schematic of the coupling between buoyancy shutdown and frictional spindown over a sloping bottom. A secondary circulation arises from the convergence and divergence of the Ekman transport corresponding to the laterally sheared mean flow $f>0$. The dashed lines represent the upper limits of the Ekman layer $\delta_{e}$ and the thermal boundary layer $\delta_{T}$; gray lines denote buoyancy contours in the thermal boundary layer; red and blue curves denote positive and negative buoyancy distribution. Ekman suction advects buoyant fluid toward the bottom boundary and Ekman pumping injects dense fluid upward. Ekman suction is stronger than Ekman pumping due to the higher-order nonlinear vertical buoyancy advection (BT13). The asymmetry in vertical buoyancy advection produces steeper isopycnal tilting on the anticyclonic flank of the along-slope jet.

The cross-slope direction $y$ increases off shore, while the along-slope direction $x$ increases with the shallower water to the right. The flow is symmetric in the alongslope direction. The angle of rotation is small $(\theta \leq 0.03)$ so that the small angle approximation $\cos \theta \approx 1$ and $\sin \theta \approx \theta$ can be applied; this approximation is valid for most oceanic continental slopes. Buoyancy $b$ here represents the density perturbation to the constant background stratification $\rho=\rho_{0}+\bar{\rho}(\hat{z})-\left(\rho_{0} / g\right) b$, where the hat represents the local vertical direction. Viscosity $\nu$ and diffusivity $\kappa$ are assumed to be constant and to have the same magnitude, so the Prandtl number $\sigma=\nu / \kappa=1$. The basic system is described by the following equations: 


$$
\begin{aligned}
\frac{\partial u}{\partial t} & +v \frac{\partial u}{\partial y}+w \frac{\partial u}{\partial z}-f(v+w \theta) \\
& =\nu\left(\frac{\partial^{2} u}{\partial y^{2}}+\frac{\partial^{2} u}{\partial z^{2}}\right) \\
\frac{\partial v}{\partial t} & +v \frac{\partial v}{\partial y}+w \frac{\partial v}{\partial z}+f u \\
& =-\frac{1}{\rho_{0}} \frac{\partial p}{\partial y}-\theta b+\nu\left(\frac{\partial^{2} v}{\partial y^{2}}+\frac{\partial^{2} v}{\partial z^{2}}\right) \\
\frac{\partial w}{\partial t} & +v \frac{\partial w}{\partial y}+w \frac{\partial w}{\partial z}+f u \theta \\
& =-\frac{1}{\rho_{0}} \frac{\partial p}{\partial z}+b+\nu\left(\frac{\partial^{2} w}{\partial y^{2}}+\frac{\partial^{2} w}{\partial z^{2}}\right) \\
\frac{\partial b}{\partial t} & +v\left(\frac{\partial b}{\partial y}-N^{2} \theta\right)+w\left(\frac{\partial b}{\partial z}+N^{2}\right) \\
& =\kappa\left(\frac{\partial^{2} b}{\partial y^{2}}+\frac{\partial^{2} b}{\partial z^{2}}\right), \quad \text { and } \\
\frac{\partial v}{\partial y}+\frac{\partial w}{\partial z} & =0
\end{aligned}
$$

Here, $\mathbf{u}=(u, v, w)$ is the velocity vector, and $N^{2}=-g \rho_{0}^{-1} \partial \bar{\rho} / \partial \hat{z}$.

The boundary conditions for the semi-infinite vertical domain are no slip, no normal flow and no normal buoyancy flux at the bottom boundary, written as

$$
\begin{cases}u=v=w=0, & z=0 \\ \frac{\partial b}{\partial z}+N^{2}=0, & z=0\end{cases}
$$

The far-field boundary conditions are

$$
\begin{cases}u \rightarrow u_{\infty}(t, y), & z \rightarrow \infty \\ v, w, b \rightarrow 0, & z \rightarrow \infty\end{cases}
$$

The along-slope mean flow $u(t, y)$ is assumed to oscillate following

$$
u_{\infty}(t, y)=U \cos (\omega t)\left[\frac{1+\cos (y / L)}{2}\right], \quad-\pi L<y<\pi L,
$$

where $\omega$ is the oscillation frequency.

There are a number of important time scales in this problem. These include the buoyancy shutdown time scale $\mathscr{T}_{\text {shutdown }}=\sigma^{-1} S^{-2} f^{-1}$, which is valid for $\sigma \sim O(1)$ and $S \ll 1(\mathrm{BT} 12)$, where $S=(N \tan \theta / f)^{2}$ is the slope Burger number (MacCready and Rhines 1991), or using the small angle approximation $S \approx(N \theta / f)^{2}$. The stratified spindown time scale is given by $\mathscr{T}_{\text {spindown }}=E^{-1 / 2} f^{-1}$, where $E=2 \nu / f H_{p}^{2}$ is the Ekman number, $H_{p}=f L / N$ is the Prandtl depth, and $\delta_{e}$ is the Ekman depth, defined below. The inertial and diffusive time scales are expressed as $\mathscr{T}_{\text {inertial }}=2 \pi f^{-1}$ and $\mathscr{T}_{\text {diffusive }}=E^{-1} f^{-1}$. The Ekman layer and the diffusive thermal boundary layer have thicknesses that can be expressed as $\delta_{e}=(2 \nu \mathscr{T})^{1 / 2}$ and $\delta_{T}=(2 \kappa \mathscr{T})^{1 / 2}$, respectively, where $\mathscr{T}$ is the appropriate characteristic time scale. This time scale need not be the same for $\delta_{e}$ and $\delta_{T}$. Our choice of time scales in determining the boundary layer thicknesses is dependent on the forcing frequency $\omega / f$, as discussed in section 3 .

The other nondimensional parameters that are relevant for this study include the Rossby number Ro = $U / f L$ and $\beta$, which is defined by the ratio of the spindown time scale to the buoyancy shutdown time scale (BT13),

$$
\beta=\frac{\mathscr{T}_{\text {spindown }}}{\mathscr{T}_{\text {shutdown }}}=S^{2} E^{-1 / 2},
$$

such that when $\beta \rightarrow 0$, buoyancy shutdown occurs over a longer time compared to the frictional spindown. Note that $\beta=0$ for the case of a flat bottom. The regime in which our simulations sit corresponds to

$$
\mathscr{T}_{\text {diffusive }} \gg \mathscr{T}_{\text {shutdown }} \sim \mathscr{T}_{\text {spindown }} \gg f^{-1} .
$$

The equivalence between $\mathscr{T}_{\text {shutdown }}$ and $\mathscr{T}_{\text {spindown }}$ is a result of our choice of $\beta=O(1)$, which is consistent with typical ocean parameters (we relax this constraint in our parameter exploration section). To these time scales we add the forcing time scale $\omega^{-1}$.

\section{Frequency regimes}

Our analysis of the boundary layer response to an oscillating mean flow is separated into three regimes based on $\omega$. The regimes are distinguished by the characteristic time scale used to nondimensionalize (1)-(8). The length, velocity, buoyancy, and pressure scales remain unchanged across the regimes and are given by

$$
\begin{aligned}
& y=L y^{\prime}, \quad z=H_{p} z^{\prime}, \\
& u=U u^{\prime}, \quad v=U v^{\prime}, \quad w=U \Gamma w^{\prime}, \quad \text { and } \\
& b=N^{2} H_{p} b^{\prime}, \quad p=\rho_{0} N^{2} H_{p}^{2} p^{\prime},
\end{aligned}
$$

where $L$ and $U$ are the characteristic lateral length scale and the along-slope velocity scale. The Prandtl depth $H_{p}$ characterizes the height above bottom to which the secondary circulation can penetrate in a stratified fluid. The background mean density is $\rho_{0}$, 

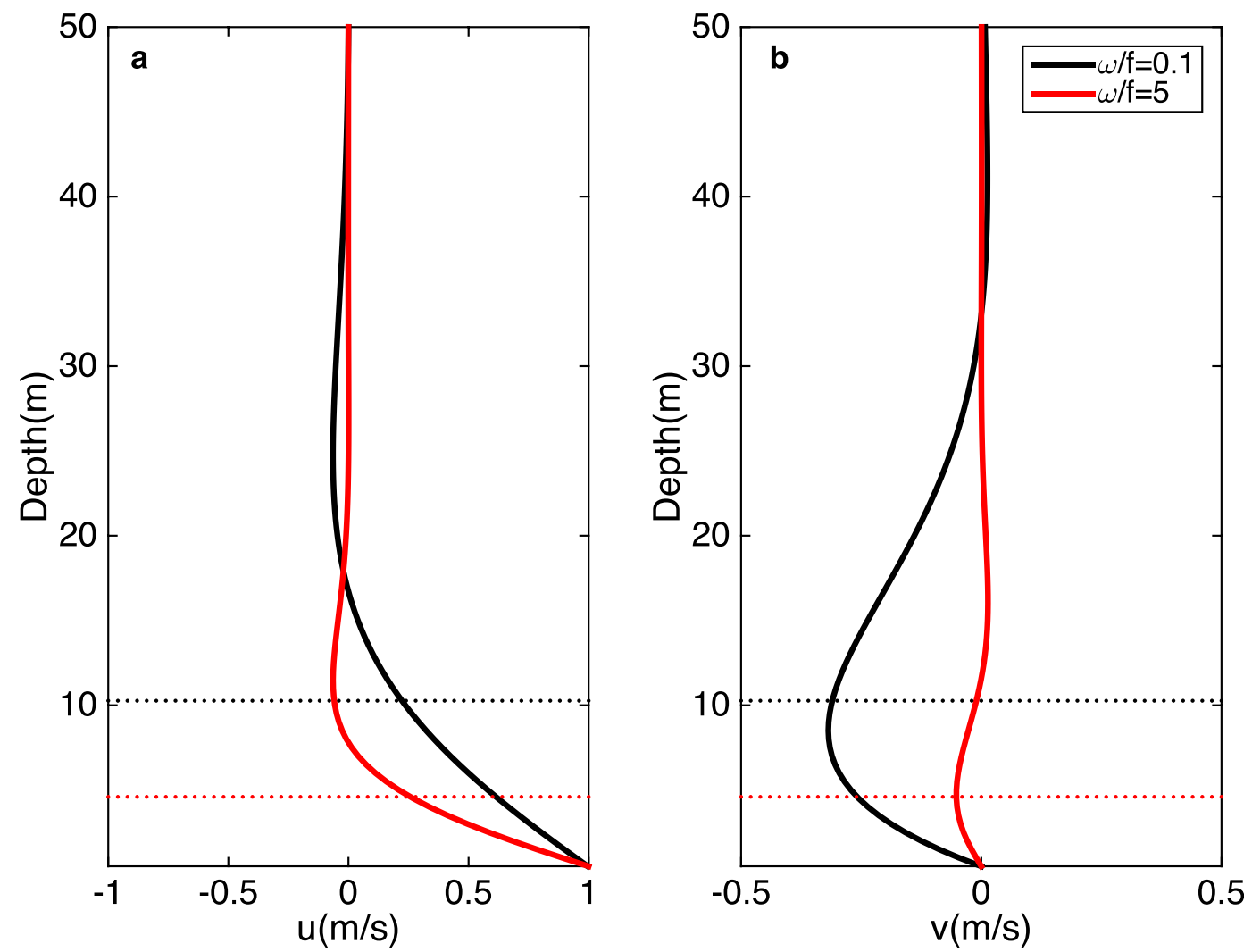

FIG. 3. Snapshots of (a) along-slope and (b) cross-slope Ekman velocities $\left(\mathrm{m} \mathrm{s}^{-1}\right)$ for a far-field boundary condition that oscillates as $\cos (\omega t)$ over a flat bottom with $\omega / f=0.1$ (black) and 0.5 (red); $f$ and $\nu$ used in this simulation are $1 \times 10^{-4} \mathrm{~s}^{-1}$ and $5.27 \times 10^{-3} \mathrm{~m}^{2} \mathrm{~s}^{-1}$, respectively. The dashed lines denote the boundary layer depth defined as $\delta=\sqrt{2 \nu / f}$ (black) and $\delta=\sqrt{2 \nu / \omega}$ (red). For $\omega / f \ll 1$, the boundary layer is arrested by Earth's rotation. For $\omega / f>1$, the Ekman layer is not well developed and cross-slope velocities are weak.

and $\Gamma=H_{p} / L$ is the aspect ratio. Based on these scales, the no buoyancy flux boundary condition and the along-slope mean flow $u(t, y)$ are nondimensionalized as (primes dropped)

$$
\begin{aligned}
& \frac{\partial b}{\partial z}+1=0, \quad z=0, \quad \text { and } \\
& u_{\infty}(t, y)=\cos (\omega t)\left[\frac{1+\cos (y)}{2}\right], \quad-\pi<y<\pi .
\end{aligned}
$$

The rest of the boundary conditions retain the same form.

In this study, we find that the time-variable mean flow has the greatest impact in the frictional Ekman layer, and variations here propagate into the thermal boundary layer and the interior via the secondary circulation. To illustrate the importance of the forcing frequency on the Ekman velocities, we solve the linear, onedimensional, time-dependent Ekman system with an oscillating, far-field boundary condition and a no-slip condition at the bottom:

$$
\frac{\partial u_{e}}{\partial t}-f v_{e}=\nu \frac{\partial^{2} u_{e}}{\partial z^{2}} ; \quad \frac{\partial v_{e}}{\partial t}+f u_{e}=\nu \frac{\partial^{2} v_{e}}{\partial z^{2}}
$$

These solutions are provided primarily to contrast with BT12 in which the Ekman layer reaches a steady state. These solutions are solved using a flat bottom, but in section 4 our full simulations are all carried out over a sloping bottom. When the forcing frequency $\omega$ is small compared to $f$, a bottom Ekman layer develops (Fig. 3, black curve) with the classic Ekman spiral in the vertical. The boundary layer thickness scales as $\delta_{e}=\sqrt{2 \nu / f}$. Thus, in the low-frequency regime $(\omega / f \ll 1)$, we assume the Ekman layer is always fully developed, even though it evolves in time. At higher frequencies, and particularly for $\omega / f \gg 1$, rotation is not important and the boundary layer scales as $\sqrt{2 \nu / \omega}<\delta_{e}$ (Fig. 3, red curve).

The following subsections show, for completeness, the nondimensionalization and decomposition of the equations of motion in the different frequency regimes. Readers primarily interested in the main results may prefer to skip to section 4. 


\section{a. The buoyancy shutdown regime: $\omega / f \ll 1$}

If the oscillation frequency is small compared to the inertial frequency $(\omega / f \ll 1)$, the characteristic time scale of the system is the frictional spindown time scale so that $t=\mathscr{T}_{\text {spindown }} t^{\prime}$. The Ekman layer thickness $\delta_{e}$ is given by $\sqrt{2 \nu / f}=E^{1 / 2} H_{p}$. The expression for $\delta_{e}$ does not involve $\mathscr{T}_{\text {spindown }}$ because the diffusion of momentum through the Ekman layer is arrested by Earth's rotation before the spindown time scale. However, the diffusive adjustment of buoyancy is not confined by rotation and the thermal boundary layer thickness is given by $\delta_{T}=$ $\left(2 \kappa \mathscr{T}_{\text {spindown }}\right)^{1 / 2}=E^{1 / 4} H_{p}>\delta_{e}$.

The nondimensionalized governing equations, with primes dropped for the dimensionless variables, are as in BT13:

$$
\begin{aligned}
& E^{1 / 2} \frac{\partial u}{\partial t}+\operatorname{Ro}\left(v \frac{\partial u}{\partial y}+w \frac{\partial u}{\partial z}\right)-(v+w \Gamma \theta) \\
& =\frac{E}{2}\left(\Gamma^{2} \frac{\partial^{2} u}{\partial y^{2}}+\frac{\partial^{2} u}{\partial z^{2}}\right) \\
& E^{1 / 2} \frac{\partial v}{\partial t}+\operatorname{Ro}\left(v \frac{\partial v}{\partial y}+w \frac{\partial v}{\partial z}\right)+u \\
& =-\operatorname{Ro}^{-1} \frac{\partial p}{\partial y}-\operatorname{Ro}^{-1} S^{1 / 2} b+\frac{E}{2}\left(\Gamma^{2} \frac{\partial^{2} v}{\partial y^{2}}+\frac{\partial^{2} v}{\partial z^{2}}\right), \\
& \operatorname{Ro} E^{1 / 2} \frac{\partial w}{\partial t}+\operatorname{Ro}^{2} \Gamma^{2}\left(v \frac{\partial w}{\partial y}+w \frac{\partial w}{\partial z}\right)+\operatorname{Ro} S^{1 / 2} \Gamma^{2} u \\
& =-\frac{\partial p}{\partial z}+b+\frac{E}{2} \operatorname{Ro}^{2}\left(\Gamma^{2} \frac{\partial^{2} w}{\partial y^{2}}+\frac{\partial^{2} w}{\partial z^{2}}\right), \\
& E^{1 / 2} \frac{\partial b}{\partial t}+\operatorname{Ro}\left(v \frac{\partial b}{\partial y}-v S^{1 / 2}\right)+\operatorname{Ro}\left(w \frac{\partial b}{\partial z}+w\right) \\
& =\frac{E}{2}\left(\Gamma^{2} \frac{\partial^{2} b}{\partial y^{2}}+\frac{\partial^{2} b}{\partial z^{2}}\right), \quad \text { and } \\
& \frac{\partial v}{\partial y}+\frac{\partial w}{\partial z}=0 .
\end{aligned}
$$

Following BT13, the nondimensionalized velocities, buoyancy, and pressure can be decomposed into three components representing the interior, thermal boundary layer, and Ekman layer, denoted by subscripts $i, T$, and $e$, respectively:

$$
\begin{aligned}
u & =u_{i}+\beta^{1 / 2} u_{T}+u_{e} \\
v & =E^{1 / 2} v_{i}+\beta^{1 / 2} E^{1 / 2} v_{T}+v_{e} \\
w & =E^{1 / 2} w_{i}+\beta^{1 / 2} E^{3 / 4} w_{T}+E^{1 / 2} w_{e} \\
b & =\operatorname{Ro} b_{i}+\operatorname{Ro} \beta^{1 / 2} S^{-1 / 2} b_{T}+\operatorname{Ro} S^{1 / 2} b_{e}, \quad \text { and } \\
p & =\operatorname{Ro} p_{i}+\operatorname{Ro} S^{1 / 2} p_{T}+\operatorname{Ro} S^{1 / 2} E^{1 / 2} p_{e}
\end{aligned}
$$

We also use a perturbation approach to solving (17) through (21), expanding to $O\left(E^{-1 / 4} \mathrm{Ro}\right)$. A key result of this previous study is that there is a coupling between the buoyancy shutdown mechanism and the interior spindown process, which causes the region of vertical Ekman suction (downwelling) to have larger amplitude than the region of Ekman pumping (upwelling). This result is achieved by considering both leading- and higher-order terms in the expansion.

\section{b. The near-inertial regime: $\omega / f \sim O(1)$}

For the low-frequency regime discussed in the previous section, the Ekman layer is in a quasi-steady state and the time tendency term in (17) through (20) is neglected. However, when $\omega$ is comparable to the inertial frequency $(\omega / f \approx 1)$, the quasi-steady state Ekman velocities are no longer valid. Furthermore, in this near-inertial regime, the response of velocities in the interior, thermal, and frictional boundary layers may exhibit different phase lags, as compared to the low-frequency regime.

Instead of the spindown time scale that characterizes the low-frequency regime, the system now responds to the shorter time scale associated with the mean flow oscillation. This modifies our previous nondimensionalization by introducing $t=t^{\prime} / \omega$, which requires the introduction of a new parameter that represents the ratio of the mean flow oscillation frequency $\omega$ and the inertial frequency $\alpha=\omega / f$. Note that the Prandtl depth remains as the vertical length scale in the nondimensionalization, as in the low-frequency regime. The Ekman and thermal boundary layer scalings also remain unmodified.

The new nondimensionalized equations are given by (primes dropped)

$$
\begin{aligned}
& \alpha \frac{\partial u}{\partial t}+\operatorname{Ro}\left(v \frac{\partial u}{\partial y}+w \frac{\partial u}{\partial z}\right)-(v+w \Gamma \theta) \\
& =\frac{E}{2}\left(\Gamma^{2} \frac{\partial^{2} u}{\partial y^{2}}+\frac{\partial^{2} u}{\partial z^{2}}\right), \\
& \alpha \frac{\partial v}{\partial t}+\operatorname{Ro}\left(v \frac{\partial v}{\partial y}+w \frac{\partial v}{\partial z}\right)+u \\
& =-\operatorname{Ro}^{-1} \frac{\partial p}{\partial y}-\operatorname{Ro}^{-1} S^{1 / 2} b+\frac{E}{2}\left(\Gamma^{2} \frac{\partial^{2} v}{\partial y^{2}}+\frac{\partial^{2} v}{\partial z^{2}}\right),
\end{aligned}
$$

$\operatorname{Ro} \Gamma^{2} \alpha \frac{\partial w}{\partial t}+\operatorname{Ro}^{2} \Gamma^{2}\left(v \frac{\partial w}{\partial y}+w \frac{\partial w}{\partial z}\right)+\operatorname{Ro}^{1 / 2} \Gamma^{2} u$

$$
\begin{gathered}
=-\frac{\partial p}{\partial z}+b+\frac{E}{2} \operatorname{Ro} \Gamma^{2}\left(\Gamma^{2} \frac{\partial^{2} w}{\partial y^{2}}+\frac{\partial^{2} w}{\partial z^{2}}\right) \\
\alpha \frac{\partial b}{\partial t}+\operatorname{Ro}\left(v \frac{\partial b}{\partial y}-v S^{1 / 2}\right)+\operatorname{Ro}\left(w \frac{\partial b}{\partial z}+w\right) \\
=\frac{E}{2}\left(\Gamma^{2} \frac{\partial^{2} b}{\partial y^{2}}+\frac{\partial^{2} b}{\partial z^{2}}\right), \text { and }
\end{gathered}
$$$$
\frac{\partial v}{\partial y}+\frac{\partial w}{\partial z}=0 .
$$ 
These equations differ from (17) to (21) in the tendency term where $\alpha$ replaces $E^{1 / 2}$. In the near-inertial regime, $\alpha$ is $O(1)$, which primarily impacts the Ekman layer, as discussed below. In the thermal boundary layer, the leading-order balance takes the form

$$
\alpha \frac{\partial b_{T}}{\partial t} \approx \frac{E}{2}\left(\frac{\partial^{2} b_{T}}{\partial z^{2}}\right)
$$

We apply a multiple-scale solution of the form $b_{T}(t, \tau)$, where $\tau=\varepsilon t$ and $\varepsilon=\left(\omega \mathscr{T}_{\text {spindown }}\right)^{-1}$. Removing the secular term at leading order results in a solution for the leading-order $b_{T}$ that adjusts diffusively on the slow time scale (as opposed to $\omega^{-1}$ ) with the same boundary layer thickness as in the low-frequency regime.

Following a similar procedure to BT13, the variable decompositions, with the new parameter $\alpha$, now become

$$
\begin{aligned}
& u=u_{i}+\alpha^{-1 / 2} S E^{-1 / 4} u_{T}+u_{e}, \\
& v=\alpha^{-1 / 2} E^{1 / 2} v_{i}+\alpha^{-1 / 2} S E^{1 / 4} v_{T}+v_{e}, \\
& w=\alpha^{-1 / 2} E^{1 / 2} w_{i}+\alpha^{-1 / 2} S E^{1 / 2} w_{T}+\alpha^{-1 / 2} E^{1 / 2} w_{e}, \\
& b=\operatorname{Ro} b_{i}+\alpha^{-1 / 2} \operatorname{Ro}^{1 / 2} E^{-1 / 4} b_{T}+\alpha^{-1} \operatorname{Ro}^{1 / 2} b_{e},
\end{aligned}
$$

and

$p=\operatorname{Rop}_{i}+\alpha^{-1 / 2} \operatorname{Ro} S^{1 / 2} p_{T}+\alpha^{-3 / 2} \operatorname{Ro} S^{1 / 2} E^{1 / 2} p_{e}$.

With the new scalings, and assuming $\alpha \approx 1$, the horizontal momentum equations in the Ekman layer are

$$
\begin{aligned}
\frac{\partial u_{e}}{\partial t}-v_{e} & =\frac{1}{2} \frac{\partial^{2} u_{e}}{\partial \eta^{2}}, \quad \text { and } \\
\frac{\partial v_{e}}{\partial t}+u_{e} & =\frac{1}{2} \frac{\partial^{2} v_{e}}{\partial \eta^{2}},
\end{aligned}
$$

where $z=E^{1 / 2} \eta$ is the scaling for the vertical distance in the Ekman layer. These are the nondimensional forms of (16). A time-dependent initial boundary value problem for a viscous, incompressible fluid in a rotating coordinate was first solved by Greenspan and Howard (1963), while Brink and Lentz (2010b) showed that this system can give rise to enhanced velocities when the forcing frequency is near the inertial frequency (a resonant interaction). For this reason, the Ekman layer velocity structure in the near-inertial region differs from that depicted in Fig. 3. This resonant interaction between the adjusting Ekman layer and the background forcing can be seen in the Ekman transport (Fig. 4), and this confirms an empirically determined velocity-stress relation discussed by Brink and Lentz (2010b).

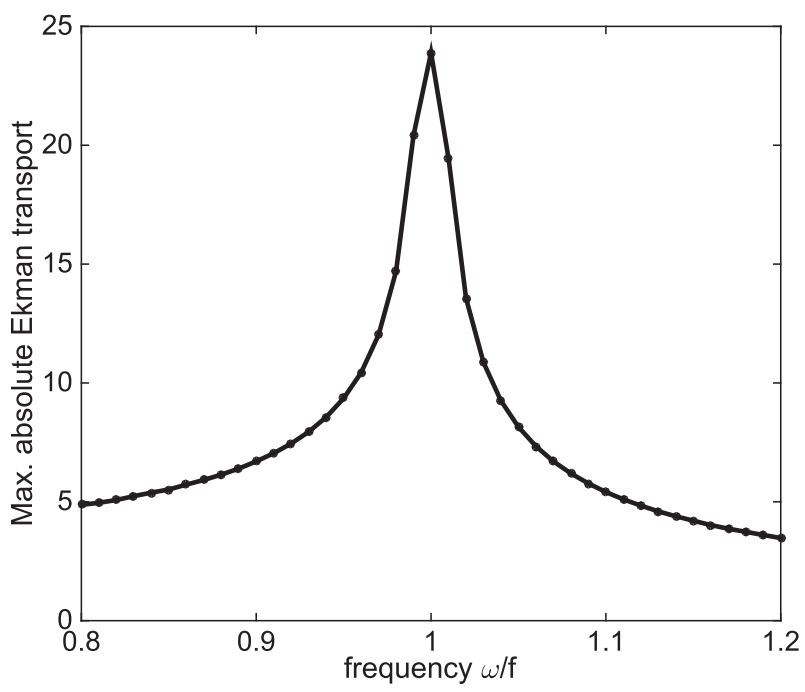

FIG. 4. Maximum (with respect to time) cross-slope Ekman transport (absolute value and nondimensionalized by $U \delta_{e}$ ) as a function of the far-field oscillation frequency $\omega$ for a onedimensional simulation. The Ekman transport is calculated as the vertical integral of the nondimensional cross-slope Ekman velocity in (38) and (39). Black dots denote individual simulations.

The scaling for the thermal boundary layer is not affected by the higher-frequency oscillations in the nearinertial regime. The buoyancy generation time scale (similar to the buoyancy shutdown and frictional spindown time scales) is still used to determine $\delta_{T}$ because diffusion dominates the distribution of buoyancy over long times. Since the buoyancy generation process arises from a competition between Ekman advection of buoyancy surfaces and their diffusive adjustment near the boundary, we can test that our choice of using $\mathscr{T}_{\text {spindown }}$ is valid by using a one-dimensional model, which has a similar setup as in BT12 but with an oscillating mean flow over a sloping bottom. The result is shown in Fig. 5. Zero buoyancy anomalies, arising from flat isopycnals are initially apparent. However, after a period comparable to the buoyancy generation (or frictional spindown) time scale, the isopycnals adjust to a state with downslope tilt, producing the persistent positive buoyancy anomalies for $t>\mathscr{T}_{\text {shutdown. The }}$ buoyancy surfaces continue to oscillate around a steady state. This solution does not include the vertical advection of buoyancy, which makes a small contribution over long time scales.

\section{c. The high-frequency regime}

For the high-frequency oscillation regime $(\omega / f \gg 1)$, the Ekman layer is no longer arrested by rotation. Instead, the frictional bottom boundary layer thickness 

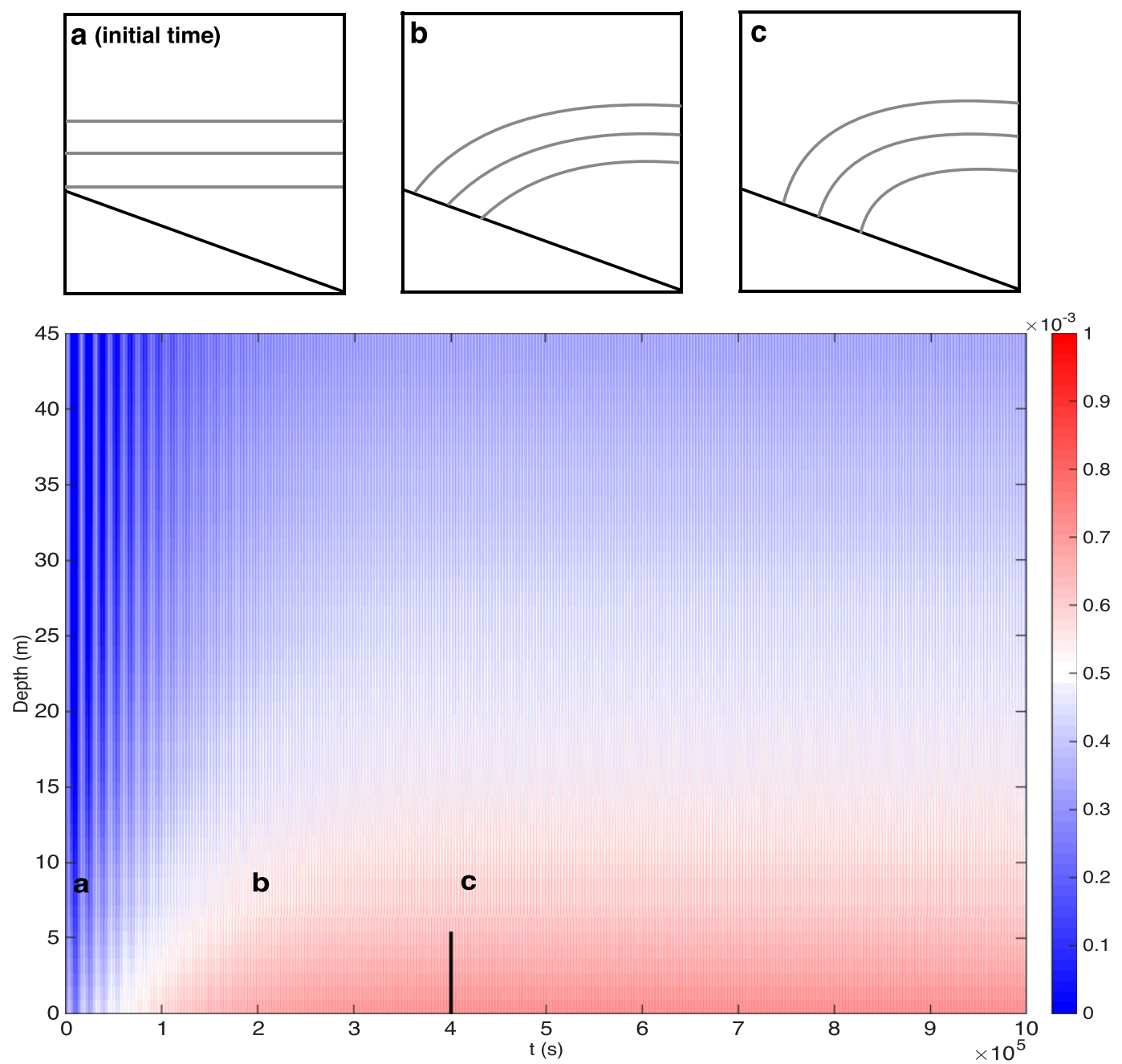

FIG. 5. Buoyancy anomalies $\left(\mathrm{m} \mathrm{s}^{-2}\right)$ over a sloping bottom $(\theta=0.01)$ in response to an oscillatory mean flow $[\sin (\omega t)]$ with frequency $\omega / f=5$. The shutdown time scale $\mathscr{T}_{\text {shutdown }}=\sigma^{-1} S^{-2} f^{-1}$ for this simulation is $4 \times 10^{5} \mathrm{~s}$ (denoted by the thick solid line). The long-term behavior is characterized by a diffusive time scale $\left(\mathscr{T}_{\text {diffusive }}=\right.$ $E^{-1} f^{-1}$ ) that tends to tilt the isopycnals downslope (positive anomalies) to meet the no-buoyancy-flux boundary condition. Schematics (a)-(c) depict the buoyancy distribution at three different times (a, b, and c in the bottom panel). Other parameters used in this simulation include $N^{2}=1.6 \times 10^{-5} \mathrm{~s}^{-2}$ and $\nu=\kappa=5.27 \times$ $10^{-3} \mathrm{~m}^{2} \mathrm{~s}^{-1}$.

can be expressed as $\delta_{e}=\sqrt{2 \nu / \omega}$. The thickness of the frictionally balanced boundary layer is dependent on the mean flow oscillation frequency, but the thermal boundary layer thickness retains the same scaling as in the near-inertial regime. For the remainder of the study, we will focus on the low-frequency and near-inertial regimes for two reasons. First, in the ocean, there are few processes that generate persistent oscillations at periods considerably smaller than the inertial period. Second, in this high-frequency regime, rotation effects are small, which leads to weak cross-slope velocities. In the absence of significant cross-slope velocities, convergence and divergence in the bottom boundary layers cannot generate strong vertical velocities and the secondary circulation will also be weak. This effect can be seen from the velocity profiles in Fig. $3 b .^{1}$

\section{Model solutions}

In the following section, we describe numerical simulations of the equations discussed in section 3. This

\footnotetext{
${ }^{1}$ Once again, the flat bottom examples discussed in this section are introduced to provide insight into the relationship between the oscillating background flow and the Ekman layer dynamics. In the remainder of this study, we discuss simulations that have a sloping bottom boundary.
} 

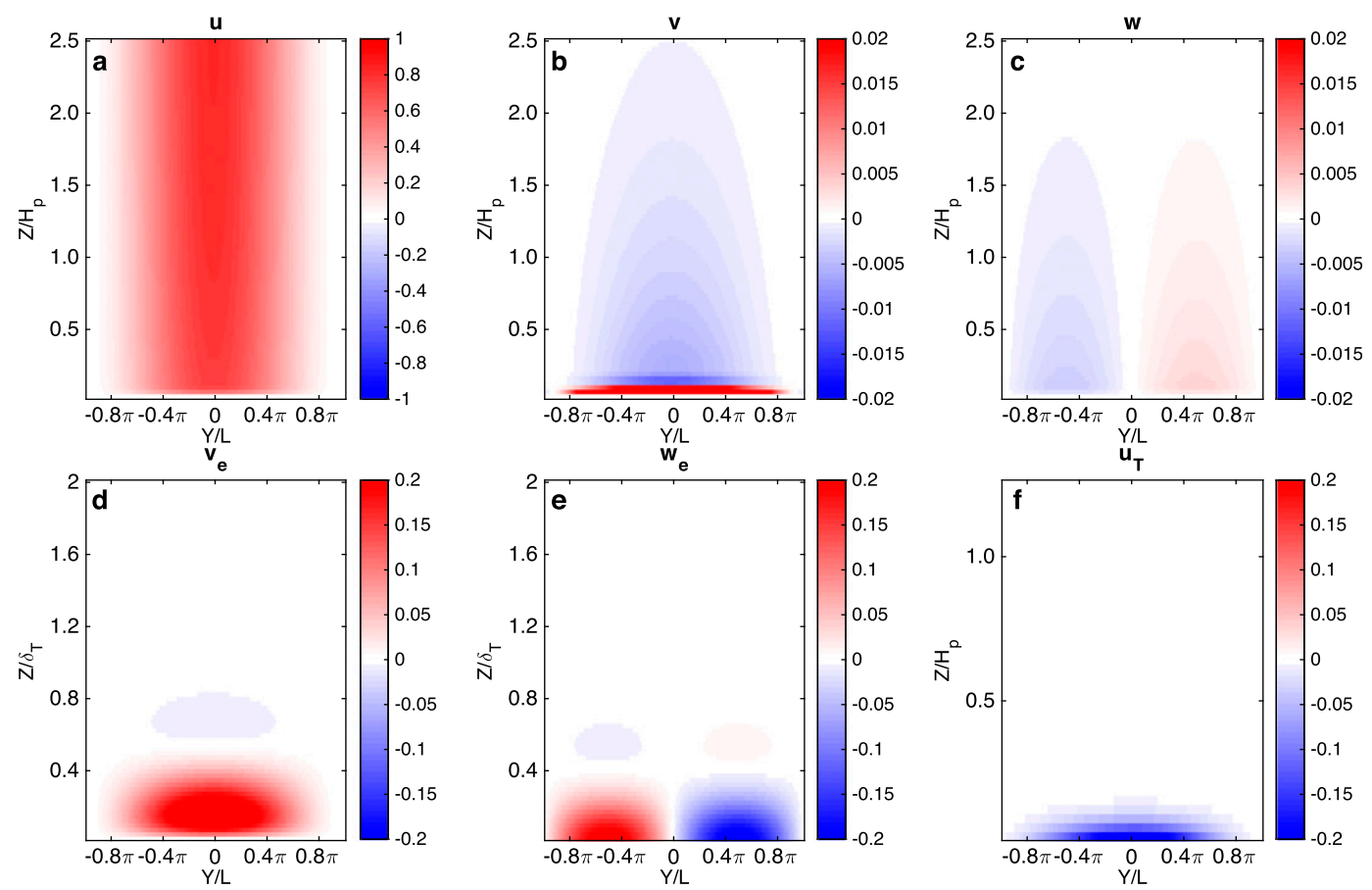

FIG. 6. Snapshots of nondimensional velocities at time $t / \mathscr{T}_{\text {spindown }}=0.15$ in the low-frequency regime $\left(\mathscr{T}_{\text {spindown }}=\right.$ $\left.E^{-1 / 2} f^{-1}\right), \omega / f=0.1$ : (a) total along-slope velocity, (b) total cross-slope velocity, (c) total velocity normal to the slope, (d) Ekman cross-slope velocity, (e) Ekman velocity normal to the slope, and (f) thermal component of the along-slope velocity. The latter is the opposing velocity from the buoyancy shutdown. Note that the vertical axes and color scales have different normalizations $\left(\delta_{T}=E^{1 / 4} H_{p}\right)$ and ranges respectively to better demonstrate the velocity structures.

section is organized similar to the previous section, with each regime discussed in a separate subsection. The equations are nondimensionalized and separated into different systems (i.e., the Ekman layer, thermal boundary layer, and interior) based on the variable decomposition given in each subsection in section 3 and then solved in each system at leading order and first order $\left(\mathrm{Ro} E^{-1 / 4}\right)$ separately using a combination of the Crank-Nicolson implicit method and Adam-Bashforth linear multistep method. Parameters that are uniform across all three regimes are the slope angle $\theta=0.01$, the jet width $L=1 \times 10^{4} \mathrm{~m}$, the Rossby number Ro $=0.1$, the Ekman number $E=6.42 \times 10^{-4}$, buoyancy frequency (squared) $N^{2}=1.6 \times 10^{-5} \mathrm{~s}^{-2}$, and the inertial frequency $f=1 \times 10^{-4} \mathrm{~s}^{-1}$. Based on these parameters, the $\beta$ value is 1.01 from (9). In the low-frequency regime, the system of equations is similar to those that appear in section 4 of BT13. In the near-inertial regime, a time-dependent Ekman layer must be added, and the revised scalings alter the expansion as detailed in the previous section.

\section{a. The buoyancy shutdown regime}

In the low-frequency regime, Ekman layer dynamics, the secondary circulation, and buoyancy diffusion are all active, which give rise to both buoyancy shutdown and frictional spindown effects. Figure 6 shows a snapshot of the velocity components from an example solution in the low-frequency regime. The snapshot is taken at $t / \mathscr{T}_{\text {spindown }}=0.15$; however, the velocity fields repeat the same cycles across each oscillation period $2 \pi / \omega$.

Although the background mean flow is continuously evolving, the inertial time scale is much shorter than the oscillation time scale, such that the Ekman layer is always close to its steady-state, developed structure. This quasi-steady Ekman transport is to the left of the mean flow $(f>0)$, as is shown in Fig. 6b. Because of the laterally sheared mean flow, convergence and divergence in the Ekman layer induce a secondary circulation that has a signature in the interior $v$ and $w$ fields (Figs. 6b,c). The component of the total vertical velocity that comes from Ekman layer dynamics $w_{e}$ has the opposite sign of the interior vertical velocity $w_{i}$, which is necessary to satisfy the bottom boundary condition $w=0$. Nonlinearity in the system arises from interaction between the buoyancy field and the vertical velocity in the thermal boundary layer. By decomposing the variables as discussed in section 3a and expanding (20) in powers of $\operatorname{Ro} E^{-1 / 4}$ (BT13), the buoyancy equation becomes

$$
\frac{\partial b_{T}}{\partial t}+\operatorname{Ro} E^{-1 / 4} w_{i}(z=0) \frac{\partial b_{T}}{\partial \xi}=\frac{1}{2} \frac{\partial^{2} b_{T}}{\partial \xi^{2}},
$$



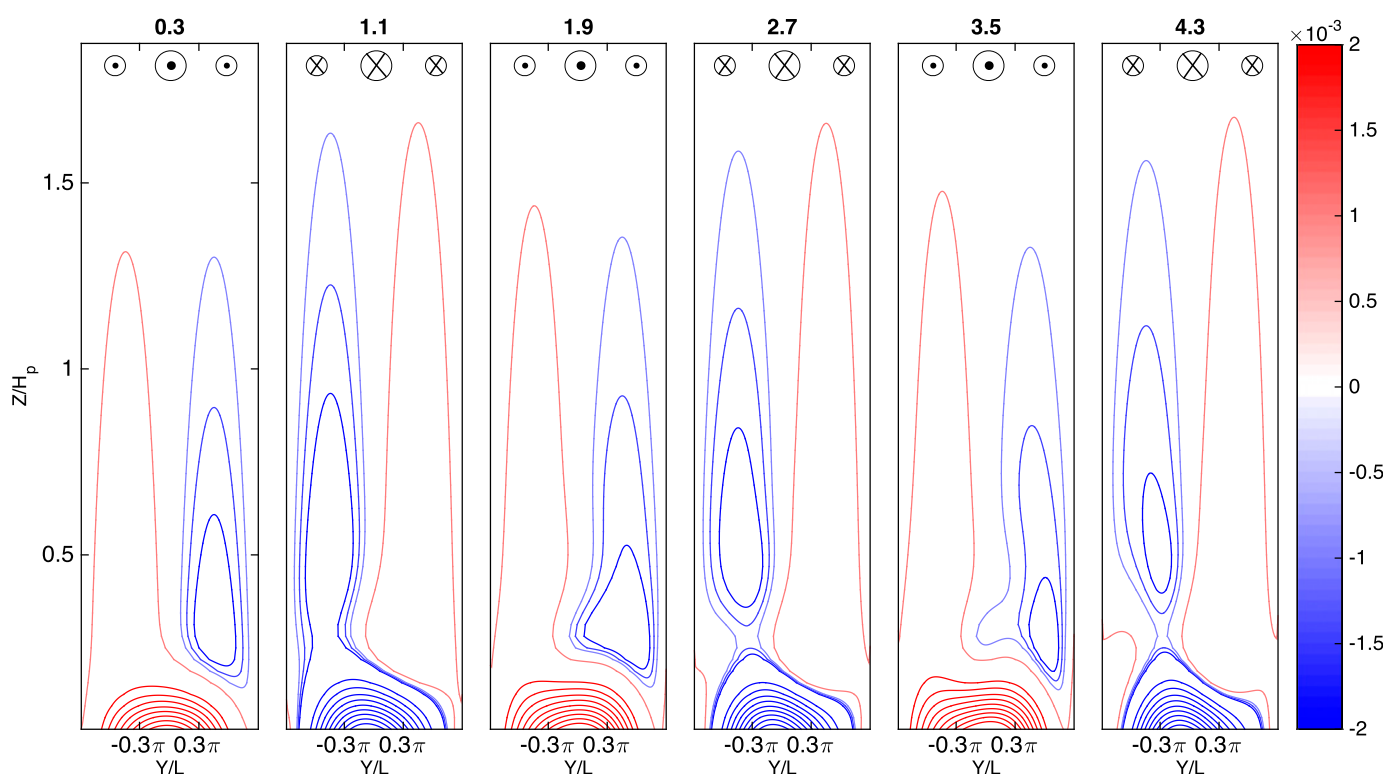

FIG. 7. Evolution of the buoyancy anomaly in the low-frequency or buoyancy shutdown regime $(\omega / f=0.1)$. Numbers above each panel denote the nondimensional time $t^{\prime}=t / \mathscr{T}$ spindown with a nondimensional period being $\pi / 2$. Snapshots are chosen to show buoyancy distributions twice per cycle of the background flow oscillation. In the Ekman and thermal boundary layers, buoyancy anomalies are generated by upslope and downslope advection of the isopycnals and buoyancy diffusion. Higher-order nonlinear vertical buoyancy advection results in slightly stronger Ekman suction compared with Ekman pumping due to stronger isopycnal tilting over the cyclonic flank of the jet.

where $\xi$ is the vertical axis for the thermal boundary layer. The vertical velocity $w_{i}$ is evaluated at the bottom since variations in $w$ across the thermal boundary layer are small.

The temporal evolution of the buoyancy field $b$ is shown in Fig. 7. The Ekman transport advects buoyancy anomalies (isopycnals) upslope and downslope in the bottommost frictional boundary layer $\delta_{e}$, and buoyancy anomalies are then diffused deeper into the thermal boundary layer $\delta_{T}$. At early times, Ekman pumping and suction are stronger during the upslope transport phase as compared to the downslope transport phase. This occurs because the fluid becomes more stratified with upslope buoyancy advection, whereas downslope buoyancy advection destroys the stratification. This asymmetry weakens over time as buoyancy diffusion takes over. At longer times, an asymmetry in the vertical extent of the buoyancy anomalies develops with positive buoyancy anomalies extending further into the interior. This arises due to the nonlinear vertical buoyancy advection in (40). As buoyancy anomalies accumulate in the interior over time, this accumulation results in an asymmetry in the isopycnal tilting with greater tilting on the cyclonic side of the along-slope current, where the negative buoyancy anomalies are located (Fig. 1). Because the isopycnals are tilted more steeply around the cyclonic axis, the resulting stronger thermal wind shear leads to stronger Ekman suction than Ekman pumping, as discussed in BT13. These asymmetries may contribute to the preference for anticyclonic vortex formation as found both in measurements (Garfield et al. 1999) and numerical simulations (Kurian et al. 2011).

While these simulations have many similarities with BT13, when the mean flow is allowed to oscillate, the system never completely achieves the shutdown state. Ekman velocities are persistently generated and continue to advect buoyancy surfaces throughout the duration of the simulation. This leads to the persistent generation of buoyancy anomalies that are continuously advected into the interior. The relationship between Ekman layer, thermal layer, and interior dynamics becomes more complicated in the near-inertial regime, as discussed in the next subsection.

\section{b. The near-inertial regime}

The structure of the velocity and buoyancy fields is similar in the near-inertial regime to the fields shown in Fig. 6. As discussed above, the biggest difference between the near-inertial regime and the low-frequency regime is that in the latter, the Ekman layer is always in a quasi-steady state. In the near-inertial regime a timedependent Ekman solution is required. Specifically, there may be a phase shift between the mean flow amplitude and the Ekman response, which can modify the coupling between the vertical velocity and the buoyancy 
or potential vorticity fields. This coupling between the different fields is sensitive to $\omega$, as discussed below.

Additionally, for $\omega \sim f$, resonance may occur in the Ekman layer, which strongly enhances the Ekman and vertical velocities. This behavior was discussed by Brink and Lentz (2010b) in the context of a turbulent boundary layer with an oscillating background mean flow with no lateral variations. We have repeated similar experiments, for example, one-dimensional with an oscillatory background mean flow but with a laminar background, consistent with the rest of this study. We also find a narrow peak in Ekman transport for $\omega=f$ (Fig. 4). Although this solution is calculated for a one-dimensional case, the Ekman transport, at each position along the continental slope, will scale with the strength of the mean flow, such that for a laterally sheared mean flow oscillation, the Ekman convergence and thus the secondary circulation will also have peak for $\omega=f$.

\section{c. The high-frequency regime}

Persistent oscillations with frequencies much greater than $f$ are rare in the ocean. Furthermore, for $\omega \gg f$, both the frictional boundary layer, essentially a diffusive layer for momentum, and the thermal boundary layer are controlled by viscosity and diffusivity (assumed constant in this study), respectively. More importantly, the Ekman layer shifts into a regime where its vertical extent is determined by $\omega$, as opposed to $f\left(\delta_{e} \sim \sqrt{\nu / \omega}\right)$. The Ekman transport is both suppressed and confined to narrower boundary layers, which results in a smaller velocity component in the cross-slope direction. Therefore, the high-frequency regime produces smaller vertical fluxes and smaller injection of fluid into the interior.

\section{PV dynamics}

The Ertel PV is defined as $q=\boldsymbol{\omega}_{a} \cdot \nabla B$, where $\nabla B=$ $\nabla b+N^{2} \hat{z}$ is the total buoyancy gradient and $\boldsymbol{\omega}_{a}=$ $f \hat{z}+\nabla \times \mathbf{u}$ is the absolute vorticity. The nondimensionalized PV is given by $q^{\prime}=q /\left(f N^{2}\right)$. For the remainder of this section, we remove the primes, but nondimensionalized quantities are understood and we discuss the nondimensional $\mathrm{PV}$ to $O\left(\operatorname{Ro} E^{-1 / 4}\right)$. The leading-order PV comes from the background stratification and in its nondimensional form is equal to 1 . The additional contributions to the potential vorticity come from vortex stretching, the relative vorticity and lateral buoyancy gradients, assumed to be in thermal wind balance. Using this approach, the PV is expressed as

$$
q \approx 1+\underbrace{\frac{\partial b}{\partial z}}_{q_{b}}-\underbrace{\operatorname{Ro} \frac{\partial u}{\partial y}}_{q_{\zeta}}-\underbrace{(\operatorname{Ro} \theta / \Gamma) \frac{\partial u}{\partial z}}_{q_{\mathrm{tw}}} .
$$

Modifications to the PV arising from the vertical buoyancy gradient $q_{b}$ are mainly due to buoyancy adjustment in the Ekman and the thermal boundary layers. The modification to the PV due to relative vorticity $q_{\zeta}$ is dominated by the horizontal gradient of the mean alongslope velocity. Finally, the vertical gradient of the alongslope velocity (thermal wind), which gives rise to $q_{\mathrm{tw}}$, is generated by the opposing geostrophic velocity in the boundary layer due to tilted isopycnals.

The oscillation frequency $\omega / f=0.1$ is adopted as an example to analyze the PV dynamics. The lowfrequency regime provides a clearer demonstration of the physical processes that are responsible for the rectification of the PV flux. A nonzero PV flux develops both in the boundary layers and in the interior, but the amplitude of the PV flux is significantly stronger within the thermal boundary layer (Fig. 8c). In the thermal boundary layer, the PV develops a positive anomaly for a negative along-slope flow, as in Fig. 8b. The positive PV near the bottom mainly comes from the stretching and thermal wind components, which are shown in Fig. 9. In the interior, the PV is dominated by horizontal shear of the mean flow. The vertical velocity arises from the Ekman convergence and is symmetric, to leading order, about $y / L=0$. At higher order, the amplitude of the downward vertical velocity in Fig. $8 \mathrm{a}$ is stronger due to vertical buoyancy advection and results in asymmetric isopycnal tilting.

The vertical eddy PV flux is given by $w^{\prime} q^{\prime}$, where ' represents a deviation from the spatial (cross slope and depth) mean value. In the thermal boundary layer, the PV flux obtains both positive and negative values; however, in a cross-slope average $\left\langle w^{\prime} q^{\prime}\right\rangle>0$, where \langle\rangle represents an average over $-\pi<y / L<\pi$ (Fig. 8d). In the interior, the sign of the horizontal shear is correlated with the sign of the vertical velocity due to the secondary circulation, such that the PV flux is always positive. Thus, the oscillatory mean flow generates a persistent rectified PV flux due correlations between the secondary circulation and the PV anomalies in both the boundary layer and the interior. In the interior, the eddy PV flux decays with height above bottom (Fig. 8d). In the boundary layer, the asymmetry in the eddy PV flux mainly results from the asymmetry in the isopycnal tilting between cyclonic and anticyclonic flanks of the along-slope flow. This then influences the stretching component of the PV. The spatially averaged PV flux $\left\langle w^{\prime} q^{\prime}\right\rangle$ is strongest in the thermal boundary layer and critically is positive for all phases of the oscillation. Converting from nondimensional variables back to dimensional quantities, we find that the eddy PV flux can penetrate to a depth of at least $500 \mathrm{~m}$ based on the current parameters. This may represent a large fraction 

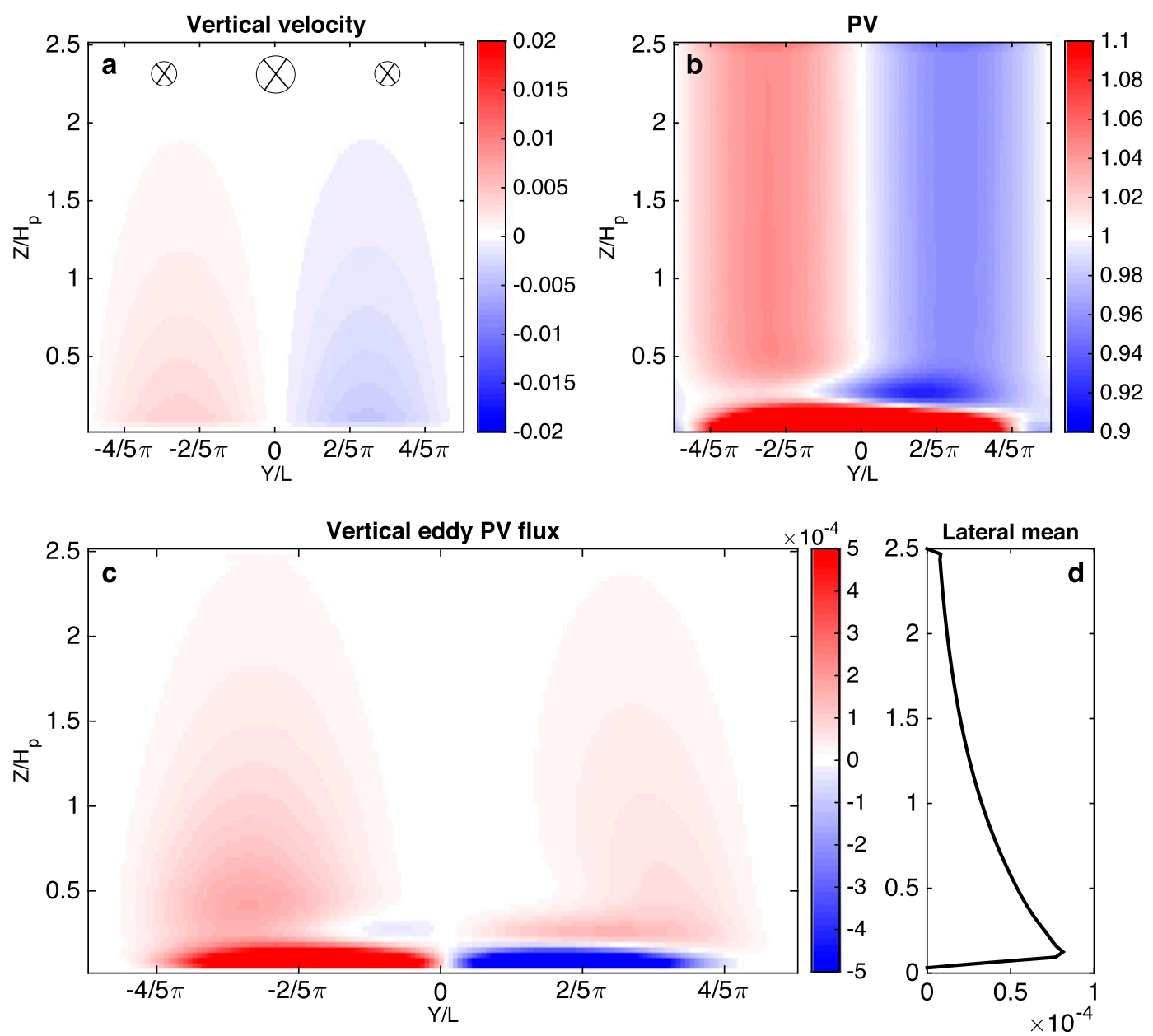

FIG. 8. Characterization of potential vorticity and vertical potential vorticity flux for an experiment with $\omega / f=0.1$ at $t / \mathscr{T}_{\text {spindown }}=2.45$ : (a) total vertical velocity, (b) Ertel PV, and (c) vertical eddy PV flux. (d) Cross-slope mean eddy PV flux corresponding to (c). The lack of cancellation of the cross-slope-averaged eddy PV flux in the bottom boundary layer results from the asymmetry in the isopycnal tilting between cyclonic and anticyclonic flanks of the jet, which directly modifies the stretching component of the PV (Fig. 9).

of the water column in regions close to the continental shelf. The PV flux has a maximum (dimensional) magnitude of order $10^{-15} \mathrm{~m} \mathrm{~s}^{-4}$, consistent with BT12 and discussed further in section 6 .

The three components of the Ertel PV anomaly, $q_{\zeta}$, $q_{b}$, and $q_{\mathrm{tw}}$, in one phase of the mean flow oscillation period, are shown in Fig. 9. Again, in the interior, the PV is dominated by $q_{\zeta}$. In the Ekman and thermal boundary layers, all three components contribute. In the Ekman layer, $q_{b}$ arises from the advection of buoyancy surfaces by the Ekman velocities (Fig. 9b). However, $q_{b}$ modifies the PV throughout the thermal boundary layer, as the buoyancy surfaces respond to vertical advection caused by Ekman convergence. This gives rise to a dipole structure in the vertical direction. In Fig. 9c, $q_{\mathrm{tw}}$ is shown for a relatively early time in the experiment. Over longer times, buoyancy diffusion will lead $q_{\mathrm{tw}}$ to extend throughout the thermal boundary layer, but it does not have the dipole structure of $q_{b}$.

Figure 10 summarizes the vertical PV flux for a range of oscillation frequencies $\omega / f$. Each dot represents a separate numerical simulation, where the eddy PV flux has been calculated as an average over time and area $(y-z$ plane $)$ after the transient adjustment period. In the low-frequency regime, the vertical eddy PV flux increases rapidly and nonlinearly with an increase in $\omega / f$ (Fig. 10, inset). In the low-frequency regime, although the Ekman layer is in a quasi-steady state, the oscillations are effective in avoiding a buoyancy shutdown scenario, which would bring the PV flux to zero (the $\omega / f=0$ case) after adjustment to the arrested state. This highlights the significance of temporal oscillations in modifying interior distributions of buoyancy and PV. As the frequency becomes larger and $\omega / f \rightarrow 1$, the near-inertial 

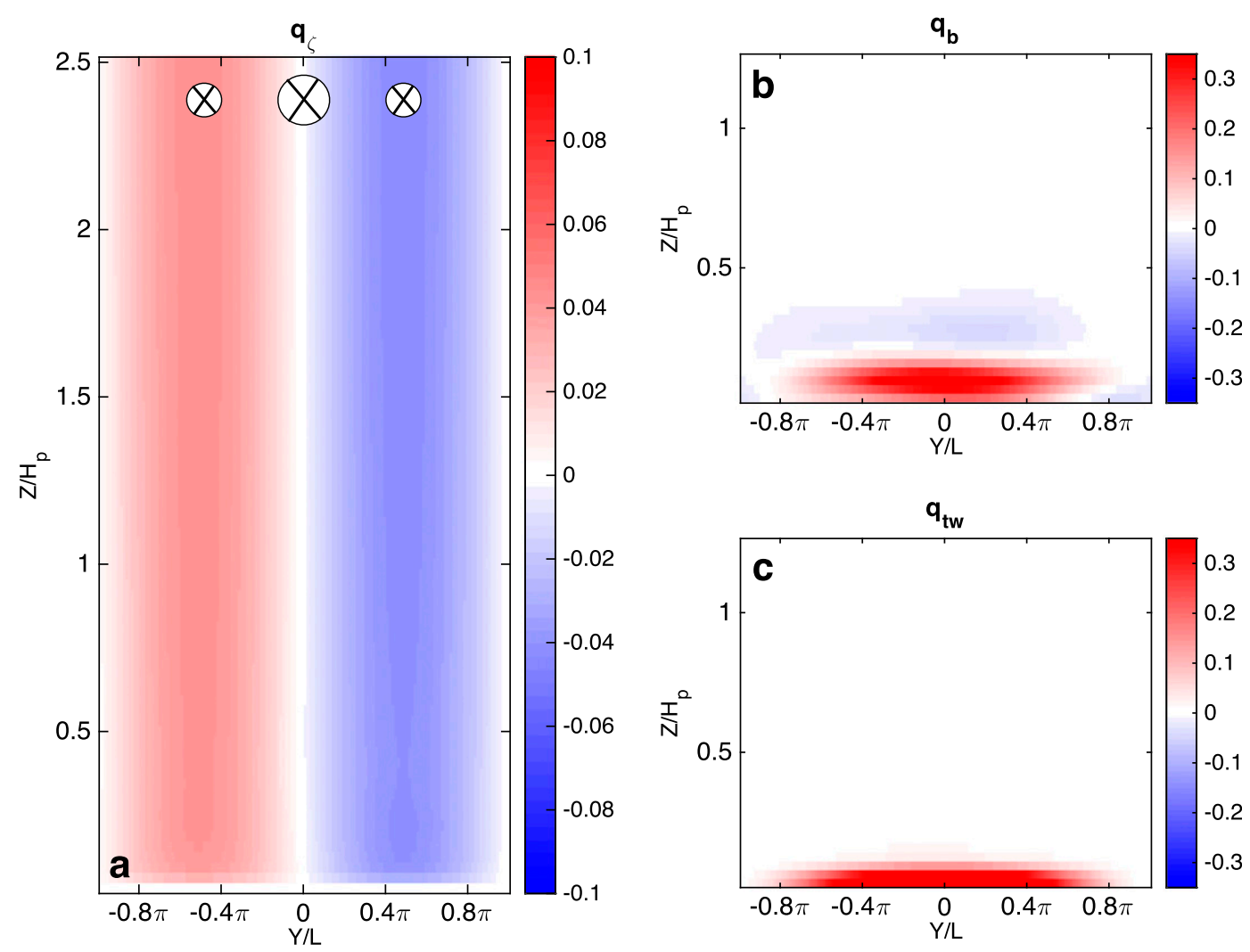

FIG. 9. Decomposition of the eddy Ertel PV for the snapshot shown in Fig. 8b: (a) $q_{\mathrm{c}}$, (b) $q_{b}$, and (c) $q_{\mathrm{tw}}$, as defined in (41). These terms represent (a) the horizontal gradient of the along-slope velocity, which is mostly from the barotropic mean flow shear throughout the water column; (b) the vertical buoyancy gradient; and (c) the vertical gradient of the along-slope velocity, which is due to the generated opposing velocity in the lower boundary. The color scales are not uniform because the vertical velocity and buoyancy gradients near the bottom boundary are larger.

regime is approached, where quasi-steady dynamics in the Ekman boundary layer is no longer expected. The phase difference between the Ekman layer and interior response would, by itself, act to reduce the PV flux. However, the resonance that occurs in the Ekman layer (Fig. 4) significantly amplifies the secondary circulation, which in turn leads to a peak in the PV flux for $\omega \approx f$. The frequency associated with the peak PV injection falls near the inertial frequency, which suggests that diurnal and semidiurnal tides, as well as inertial oscillations, may be critical for understanding water mass structure and fluid transport in coastal regions (Flexas et al. 2015). For $\omega>f$, the amplitude of the eddy PV flux decays rapidly, which can be explained by the dynamics in the high-frequency regime where there is no longer strong Ekman velocities generated. The change in sign of the PV flux for the high-frequency simulations is related to changes in the phase between oscillations in the velocity and the buoyancy anomalies. This abrupt change in sign needs to be verified in more realistic models.

An exploration of the sensitivity of the vertical PV flux to external parameters, including the mean flow amplitude, bottom slope angle, and background stratification, is shown in Fig. 11. Two values of $\omega / f$ are chosen: $\omega / f=0.1$ for the low-frequency regime and $\omega / f=0.6$ for the near-inertial regime. The Coriolis frequency is held fixed, although its influence on the simulation can be seen in terms of the slope Burger number $S=(N \theta / f)^{2}$. The model setup is the same as is presented in section 4 .

Increasing the amplitude of the background mean flow leads to stronger convergence and divergence and thus stronger vertical velocities (Fig. 11a), which results in a larger vertical PV flux. Mean flow amplitudes are tested between 0 and $0.2 \mathrm{~m} \mathrm{~s}^{-1}$, which is equivalent to a change in Ro between 0 and 0.2 , since $f L=1 \mathrm{~m} \mathrm{~s}^{-1}$. The eddy PV flux is more sensitive to the amplitude of the topographic slope in the low-frequency regime (Fig. 11b). This can be explained by (9). As the slope angle $\theta$ increases, the slope Burger number $S$ increases, leading to a more effective buoyancy shutdown, that is, it occurs over a shorter time scale. Thus, during each oscillation cycle, the Ekman layer comes closer to achieving buoyancy shutdown, which reduces the vertical velocity and limits the vertical PV flux. However, in 


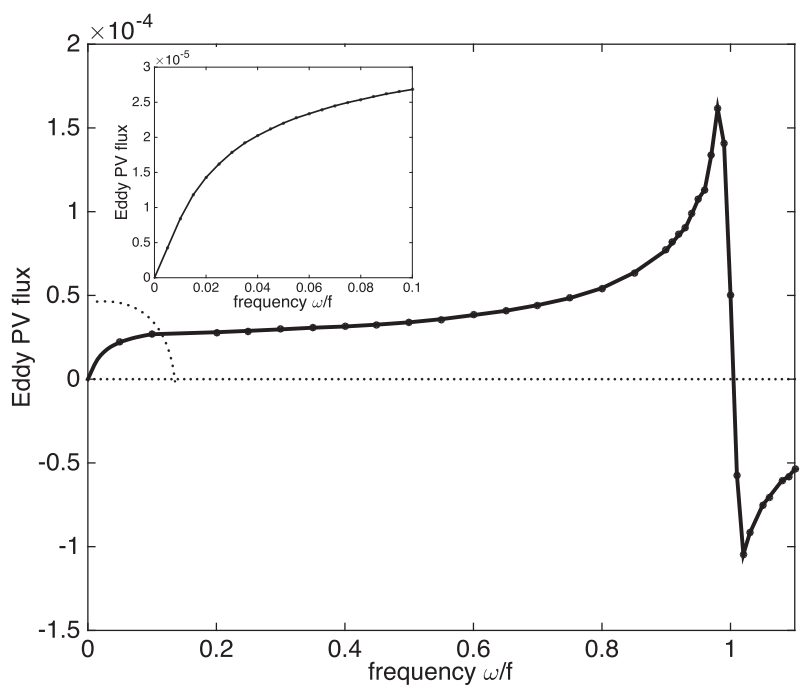

FIG. 10. Time- and area-averaged nondimensional vertical eddy PV flux $\left(\left\langle\overline{w^{\prime} q^{\prime}}\right\rangle / \Gamma U f N^{2} \operatorname{Ro} E^{-1 / 4}\right)$ as a function of oscillation frequency $\omega$. Black dots denote individual simulations. The dotted lines indicate the parameter space shown in the inset plot.

the near-inertial regime, the buoyancy shutdown effect is already weak and therefore the vertical PV flux is almost unaffected by changes in $\theta$. The PV flux increases as the stratification increases (Fig. 11c). When the stratification increases, the PV anomaly is enhanced primarily through buoyancy-related contributions and thus an enhanced eddy PV flux. Note that the PV fluxes calculated in Fig. 11c are integrated in the depth range $0<z<H_{p}$, rather than throughout the entire domain. This is done because $H_{p}$ changes with the stratification and the PV flux extends to different depths when $N^{2}$ is varied. Also, considering the variable $H_{p}$, the vertical axis in Fig. 12 is nondimensionalized using $\delta_{T}$. In summary, larger background stratification will lead to a stronger PV export out of the thermal boundary layer, but this PV flux will not penetrate as far vertically into the interior.

\section{Conclusions}

The coupling between the buoyancy shutdown and the frictional spindown processes with a laterally sheared, oscillating mean flow is explored using an asymptotic approach to solving the equations of motion. Three distinct physical regimes are identified. In the low-frequency regime, in which buoyancy shutdown is effective, Ekman transport produces buoyancy anomalies in the frictional boundary layer. The resultant vertical buoyancy gradient interacts with the Ekmaninduced vertical velocity in a thermal boundary layer; buoyancy diffusion also occurs in this layer. More importantly, the convergence and divergence in the Ekman transport generates a secondary circulation that
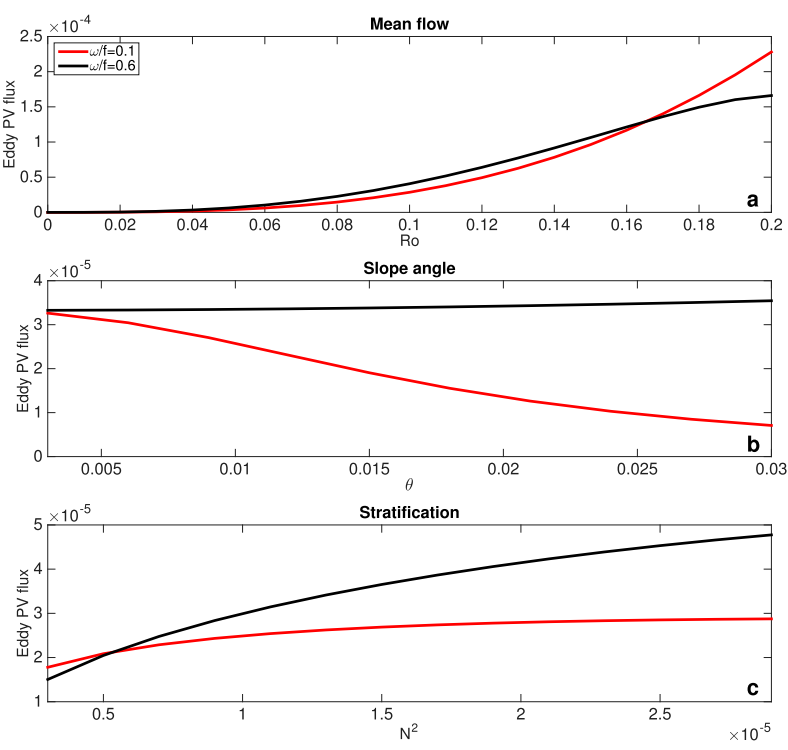

FIG. 11. Time- and area-averaged vertical eddy PV flux as a function of (a) Rossby number (b) slope angle $\theta$ and (c) background stratification $N^{2}\left(\mathrm{~s}^{-2}\right)$. The latter influences the nondimensional Ekman number $E$. In (c), the depth over which the PV flux was integrated is equivalent to the Prandtl depth $H_{p}$, since $H_{p}$ varies with changes in $N^{2}$. Red and black curves are for simulations with $\omega / f=0.1$ (low-frequency regime) and $\omega / f=0.6$ (near-inertial regime), respectively.

penetrates up to $500 \mathrm{~m}$ with realistic ocean parameters. The coupling between the vertical circulation and PV anomalies results in a rectified vertical eddy PV flux. In this study, the dominant contribution of PV anomalies is from the laterally sheared background mean flow rather than from the BBL. Detailed quantification of frictional and diabatic PV fluxes from the BBL awaits further studies using more realistic models. In the absence of oscillations, buoyancy shutdown may run its course and produce a state with no flow in the Ekman layer and no secondary circulation, which eliminates the eddy PV flux as well as the frictional and diabatic PV fluxes due to the slippery (no stress) boundary layer. Even relatively slow temporal oscillations are shown to effectively avoid this state. In the near-inertial regime, the PV injection is influenced by time-dependent Ekman dynamics. As $\omega$ approaches $f$, resonance in the Ekman layer results in amplification of the secondary circulation and a peak in the PV flux. Finally, in the high-frequency regime, narrower boundary layers and weaker cross-slope transport greatly reduce the injection of the PV anomaly into the ocean interior.

The vertical eddy PV flux depends on both the oscillation frequency and various external parameters. Specifically, the eddy PV flux is a nonlinear function of oscillation frequencies, with strong sensitivity in the low-frequency regime; the PV flux peaks when $\omega \approx f$. Mean flow amplitude directly influences the Ekman transport, the secondary 

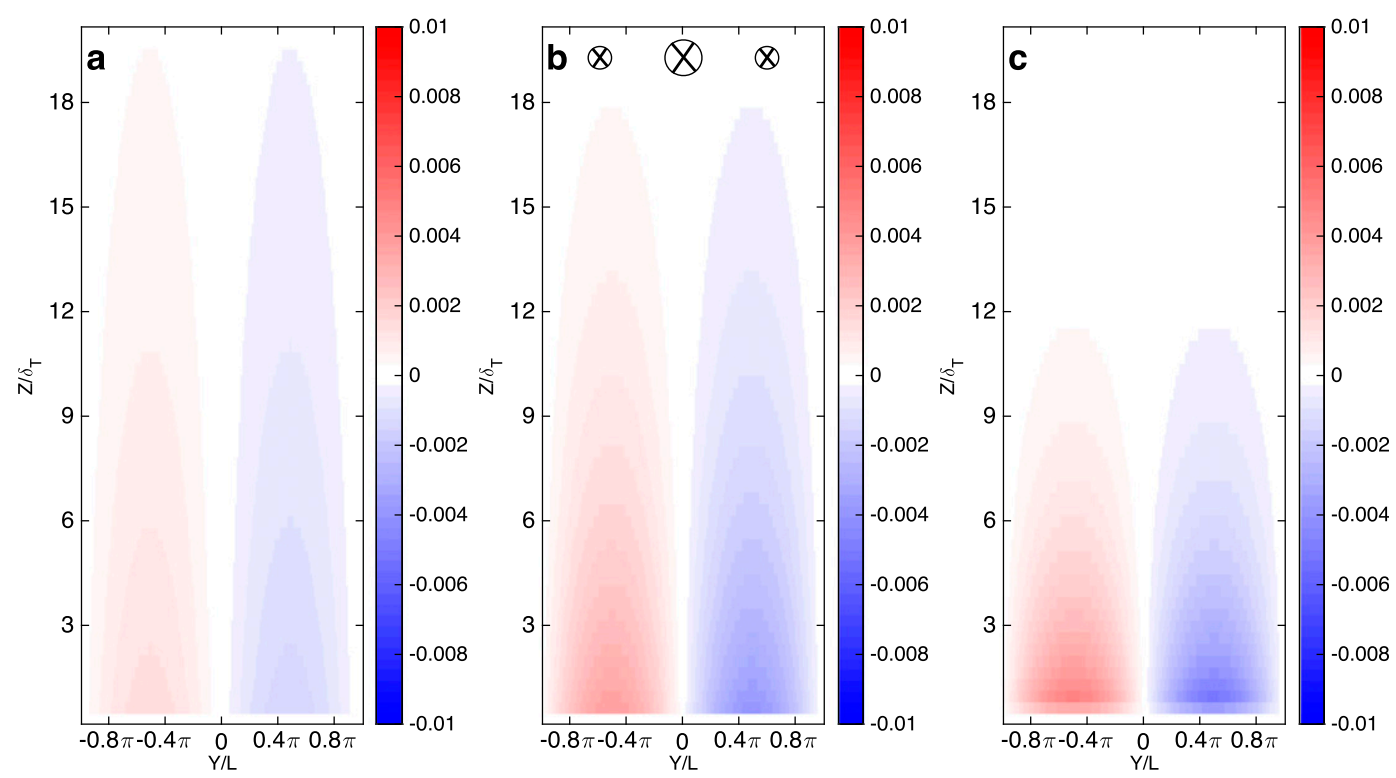

FIG. 12. Nondimensional interior vertical velocity distribution $\left(w_{i} / \Gamma U\right)$ for an experiment with $\omega / f=0.1$ at $t / \mathscr{T}_{\text {spindown }}=4.0$ and different stratifications: $N^{2}=$ (a) $1 \times 10^{-6}$, (b) $1 \times 10^{-5}$, and (c) $1 \times 10^{-4} \mathrm{~s}^{-2}$. The penetration depth of the secondary circulation decreases with increasing $N^{2}$.

circulation, and thus the PV pumping. The impact of the slope angle is more acute in the buoyancy shutdown regime where the slope angle, contained in the slope Burger number, determines the efficiency of the buoyancy shutdown process over an oscillation period. Increases in background $N^{2}$ enhance the injection of PV into the interior, but the stronger interior stratification limits the vertical extent of the eddy PV flux.

The formation of mesoscale and submesoscale eddies along the continental slope arising from interactions between mean flows and sloping topography have recently received attention in a number of studies (Molemaker et al. 2015; Gula et al. 2015). This work has emphasized continental slopes as a lateral boundary that serves as a platform for ageostrophic centrifugal instability to occur. We propose that the PV anomalies that are injected by the processes discussed in this paper could additionally influence the formation of deep eddies near continental shelves and slopes. A key result of this work is that buoyancy and PV anomalies generated in thin boundary layers may be advected much deeper into the interior through secondary circulations. Variability in boundary currents over a range of time scales is likely to minimize the importance of complete buoyancy shutdown. Future work needs to more directly address the interaction between interior and BBL flows than could be explored in the simple model considered here.

The generation of PV anomalies due to surface wind stresses, for example, downfront winds (Thomas 2005), has greatly improved our understanding of mixed layer variability and the upper-ocean PV budget. Our understanding of variability of bottom circulations is considerably more limited; this idealized study offers support for the bottom boundary layer, playing an important role in largerscale circulation dynamics. This bottom PV pump mechanism should be considered in the context of slope current variability, water mass formation, and continental shelfslope exchange. These dynamics may be particularly important in high-latitude regions where relatively warm but deep waters must cross large topographic (PV) barriers, for example, Circumpolar Deep Water at the Antarctic margins (Thoma et al. 2008; Thompson et al. 2014). The size of the PV fluxes that occur through the oscillating mean flow are small compared to PV fluxes generated at the ocean surface, (e.g., Thomas 2005) or over the shallow continental shelf (Gula et al. 2015). Nevertheless, the mechanism described here is capable of generating a persistent PV flux in the presence of a variable along-slope current, for instance due to a tidally oscillating boundary current. As mentioned by BT12, the net modification depends on both a temporal and spatial average of the PV fluxes. Surface processes are likely to be intermittent in both space and time, whereas boundary currents may extend over a broad geographic extent along continental slopes and midocean ridges. While this model is too simple to provide a global assessment of its contribution to deep-ocean PV fields, it focuses the attention of future work on boundary processes, consistent with recent assessments of the importance of sloping bottoms on the abyssal overturning circulation (Mashayek et al. 2015). 
Acknowledgments. We thank two anonymous reviewers as well as Jessica Benthuysen, Leif Thomas, Andrew Stewart, Georgy Manucharyan, and Kaushik Srinivasan for helpful comments and conversations that improved this manuscript. We gratefully acknowledge support from NSF Award OPP-1246460.

\section{REFERENCES}

Benthuysen, J., and L. N. Thomas, 2012: Friction and diapycnal mixing at a slope: Boundary control of potential vorticity. J. Phys. Oceanogr., 42, 1509-1523, doi:10.1175/JPO-D-11-0130.1.

$\longrightarrow$, and -2013 : Nonlinear stratified spindown over a slope. J. Fluid Mech., 726, 371-403, doi:10.1017/jfm.2013.231.

Brink, K. H., and S. J. Lentz, 2010a: Buoyancy arrest and bottom Ekman transport. Part I: Steady flow. J. Phys. Oceanogr., 40, 621-635, doi:10.1175/2009JPO4266.1.

$\longrightarrow$, and - 2010b: Buoyancy arrest and bottom Ekman transport. Part II: Oscillating flow. J. Phys. Oceanogr., 40, 636655, doi:10.1175/2009JPO4267.1.

Chapman, D. C., 2002: Deceleration of a finite-width, stratified current over a sloping bottom: Frictional spindown or buoyancy shutdown? J. Phys. Oceanogr., 32, 336-352, doi:10.1175/ 1520-0485(2002)032<0336:DOAFWS > 2.0.CO;2.

Dulaiova, H., M. Ardelan, P. B. Henderson, and M. A. Charette, 2009: Shelf-derived iron inputs drive biological productivity in the southern Drake Passage. Global Biogeochem. Cycles, 23, GB4014, doi:10.1029/2008GB003406.

Flexas, M., M. Schodlok, L. Padman, D. Menemenlis, and A. Orsi, 2015: Role of tides on the formation of the Antarctic Slope Front at the Weddell-Scotia Confluence. J. Geophys. Res. Oceans, 120, 3658-3680, doi:10.1002/2014JC010372.

Garfield, N., C. A. Collins, R. G. Paquette, and E. Carter, 1999: Lagrangian exploration of the California Undercurrent, 1992-95. J. Phys. Oceanogr., 29, 560-583, doi:10.1175/ 1520-0485(1999)029<0560:LEOTCU>2.0.CO;2.

Gayen, B., and S. Sarkar, 2011: Negative turbulent production during flow reversal in a stratified oscillating boundary layer on a sloping bottom. Phys. Fluids, 23, 101703, doi:10.1063/1.3651359.

,$- \ldots$, and J. R. Taylor, 2010: Large eddy simulation of a stratified boundary layer under an oscillatory current. J. Fluid Mech., 643, 233-266, doi:10.1017/S002211200999200X.

Greenspan, H., and L. Howard, 1963: On a time-dependent motion of a rotating fluid. J. Fluid Mech., 17, 385-404, doi:10.1017/ S0022112063001415.

Gruber, N., and Coauthors, 2006: Eddy-resolving simulation of plankton ecosystem dynamics in the California Current System. Deep-Sea Res. I, 53, 1483-1516, doi:10.1016/ j.dsr.2006.06.005.

Gula, J., M. J. Molemaker, and J. C. McWilliams, 2015: Gulf Stream dynamics along the southeastern U.S. seaboard. J. Phys. Oceanogr., 45, 690-715, doi:10.1175/JPO-D-14-0154.1.

Kurian, J., F. Colas, X. Capet, J. C. McWilliams, and D. B. Chelton, 2011: Eddy properties in the California Current System. J. Geophys. Res., 116, C08027, doi:10.1029/2010JC006895.

MacCready, P., and P. B. Rhines, 1991: Buoyant inhibition of Ekman transport on a slope and its effect on stratified spin-up. J. Fluid Mech., 223, 631-661, doi:10.1017/S0022112091001581.

Mashayek, A., R. Ferrari, M. Nikurashin, and W. Peltier, 2015: Influence of enhanced abyssal diapycnal mixing on stratification and the ocean overturning circulation. J. Phys. Oceanogr., 45, 2580-2597, doi:10.1175/JPO-D-15-0039.1.

Molemaker, M. J., J. C. McWilliams, and W. K. Dewar, 2015: Submesoscale instability and generation of mesoscale anticyclones near a separation of the California Undercurrent. J. Phys. Oceanogr., 45, 613-629, doi:10.1175/JPO-D-13-0225.1.

Moum, J., A. Perlin, J. Klymak, M. Levine, T. Boyd, and P. Kosro, 2004: Convectively driven mixing in the bottom boundary layer. J. Phys. Oceanogr., 34, 2189-2202, doi:10.1175/ 1520-0485(2004)034<2189:CDMITB > 2.0.CO;2.

Orsi, A. H., W. M. Smethie, and J. L. Bullister, 2002: On the total input of Antarctic waters to the deep ocean: A preliminary estimate from chlorofluorocarbon measurements. J. Geophys. Res., 107 (C8), doi:10.1029/2001JC000976.

Perlin, A., J. Moum, and J. Klymak, 2005: Response of the bottom boundary layer over a sloping shelf to variations in alongshore wind. J. Geophys. Res., 110, C10S09, doi:10.1029/2004JC002500.

Rhines, P. B., 1986: Vorticity dynamics of the oceanic general circulation. Annu. Rev. Fluid Mech., 18, 433-497, doi:10.1146/ annurev.fl.18.010186.002245.

Schneider, T., I. M. Held, and S. T. Garner, 2003: Boundary effects in potential vorticity dynamics. J. Atmos. Sci., 60, 1024-1040, doi:10.1175/1520-0469(2003)60<1024:BEIPVD>2.0.CO;2.

Spall, M. A., R. S. Pickart, P. S. Fratantoni, and A. J. Plueddemann, 2008: Western Arctic shelfbreak eddies: Formation and transport J. Phys. Oceanogr., 38, 1644-1668, doi:10.1175/2007JPO3829.1.

Thoma, M., A. Jenkins, D. Holland, and S. Jacobs, 2008: Modelling circumpolar deep water intrusions on the Amundsen Sea continental shelf, Antarctica. Geophys. Res. Lett., 35, L18602, doi:10.1029/2008GL034939.

Thomas, L. N., 2005: Destruction of potential vorticity by winds. J. Phys. Oceanogr., 35, 2457-2466, doi:10.1175/JPO2830.1.

Thompson, A. F., K. J. Heywood, S. Schmidtko, and A. L. Stewart, 2014: Eddy transport as a key component of the Antarctic overturning circulation. Nat. Geosci., 7, 879-884, doi:10.1038/ngeo2289.

Thorpe, S., 1987: Current and temperature variability on the continental slope. Philos. Trans. Roy. Soc. London, A323, 471517, doi:10.1098/rsta.1987.0100.

Trowbridge, J., and S. Lentz, 1991: Asymmetric behavior of an oceanic boundary layer above a sloping bottom. J. Phys. Oceanogr., 21, 1171-1185, doi:10.1175/1520-0485(1991)021<1171: ABOAOB $>2.0 . \mathrm{CO} ; 2$.

— northern California shelf. J. Phys. Oceanogr., 28, 2075-2093, doi:10.1175/1520-0485(1998)028<2075:DOTBBL>2.0.CO;2.

Whitworth, T., A. Orsi, S.-J. Kim, W. Nowlin, and R. Locarnini, 1998: Water masses and mixing near the Antarctic slope front. Ocean, Ice, and Atmosphere: Interactions at the Antarctic Continental Margin, S. J. Jacobs and R. F. Weiss, Eds., Antarctic Research Series, Vol. 75, Amer. Geophys. Union, 1-27.

Williams, R. G., and V. Roussenov, 2003: The role of sloping sidewalls in forming potential vorticity contrasts in the ocean interior. J. Phys. Oceanogr., 33, 633-648, doi:10.1175/ 1520-0485(2003)33<633:TROSSI $>2.0$. CO;2.

Woodgate, R. A., K. Aagaard, R. D. Muench, J. Gunn, G. Björk, B. Rudels, A. Roach, and U. Schauer, 2001: The Arctic Ocean boundary current along the Eurasian slope and the adjacent Lomonosov Ridge: Water mass properties, transports and transformations from moored instruments. Deep-Sea Res. I, 48, 1757-1792, doi:10.1016/S0967-0637(00)00091-1.

Wunsch, C., 1970: On oceanic boundary mixing. Deep-Sea Res. Oceanogr. Abstr., 17, 293-301, doi:10.1016/0011-7471(70)90022-7. 